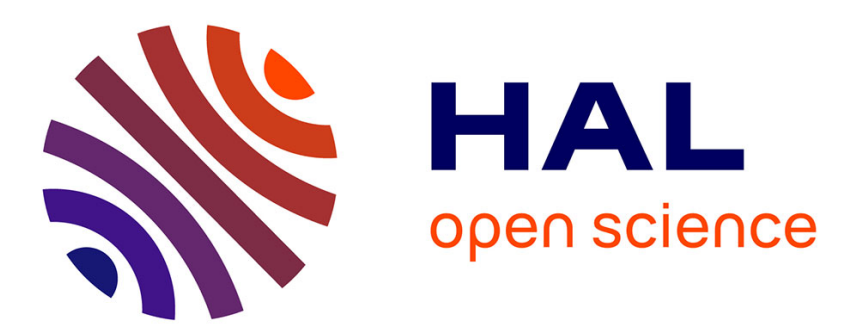

\title{
Crossover equation of state models applied to the critical behavior of Xenon
}

Yves Garrabos, Carole Lecoutre, Samuel Marre, Romain Guillaument, Daniel Beysens, Inseob Hahn

\section{- To cite this version:}

Yves Garrabos, Carole Lecoutre, Samuel Marre, Romain Guillaument, Daniel Beysens, et al.. Crossover equation of state models applied to the critical behavior of Xenon. Journal of Statistical Physics, 2015, 158 (6), pp.1379-1412. 10.1007/s10955-014-1157-x . hal-01128401

\section{HAL Id: hal-01128401 \\ https://hal.science/hal-01128401}

Submitted on 25 Jan 2021

HAL is a multi-disciplinary open access archive for the deposit and dissemination of scientific research documents, whether they are published or not. The documents may come from teaching and research institutions in France or abroad, or from public or private research centers.
L'archive ouverte pluridisciplinaire HAL, est destinée au dépôt et à la diffusion de documents scientifiques de niveau recherche, publiés ou non, émanant des établissements d'enseignement et de recherche français ou étrangers, des laboratoires publics ou privés. 


\title{
Crossover Equation of State Models Applied to the Critical Behavior of Xenon
}

\author{
Y. Garrabos - C. Lecoutre - S. Marre - R. Guillaument • \\ D. Beysens · I. Hahn
}

\begin{abstract}
The turbidity $(\tau)$ measurements of Güttinger and Cannell (Phys Rev A 24:3188$3201,1981)$ in the temperature range $28 \mathrm{mK} \leq T-T_{c} \leq 29 \mathrm{~K}$ along the critical isochore of homogeneous xenon are reanalyzed. The singular behaviors of the isothermal compressibility $\left(\kappa_{T}\right)$ and the correlation length $(\xi)$ predicted from the master crossover functions are introduced in the turbidity functional form derived by Puglielli and Ford (Phys Rev Lett 25:143-146, 1970). We show that the turbidity data are thus well represented by the Ornstein-Zernike approximant, within $1 \%$ precision. We also introduce a new crossover master model (CMM) of the parametric equation of state for a simple fluid system with no adjustable parameter. The CMM model and the phenomenological crossover parametric model are compared with the turbidity data and the coexisting liquid-gas density difference $\left(\Delta \rho_{L V}\right)$. The excellent agreement observed for $\tau, \kappa_{T}, \xi$, and $\Delta \rho_{L V}$ in a finite temperature range well beyond the Ising-like preasymptotic domain confirms that the Ising-like critical crossover behavior of xenon can be described in conformity with the universal features estimated by the renormalization-group methods. Only 4 critical coordinates of the vapor-liquid
\end{abstract}

Y. Garrabos · C. Lecoutre · S. Marre · R. Guillaument CNRS, ICMCB-ESEME, UPR 9048, 33600 Pessac, France

Y. Garrabos $(\varangle) \cdot$ C. Lecoutre · S. Marre · R. Guillaument Univ. Bordeaux, ICMCB, UPR 9048, 33600 Pessac, France e-mail: garrabos@icmcb-bordeaux.cnrs.fr

D. Beysens

ESEME-CEA, Physique et Mécanique des Milieux Hétérogènes, UMR 7636 CNRS - ESPCI - Université Pierre et Marie Curie - Université Paris Diderot, 10 rue Vauquelin, 75005 Paris, France e-mail: dbeysens@cea.fr

D. Beysens

Service des Basses Températures, UMR-E CEA/UJF-Grenoble 1, INAC, Grenoble, France

I. Hahn

Jet Propulsion Laboratory, California Institute of Technology, Pasadena, CA 91109, USA

e-mail: inseob.hahn@jpl.nasa.gov 
critical point are needed in the (pressure, temperature, molecular volume) phase surface of xenon.

Keywords Critical crossover function $\cdot$ Parametric equation of state $\cdot$ Turbidity $\cdot$ Xenon

\section{Introduction}

In their experimental paper devoted to a first unambiguous evaluation of the corrections-toscaling in the susceptibility of xenon using turbidity measurements along the critical isochore, Güttinger and Cannell (GC) [1] (reference also labeled as I herein) concluded that any theoretical progress, which would effectively reduce the number of adjustable parameters, would be extremely valuable. Moreover, it seems that the GC turbidity data are the last published data studying a critical one-component fluid with the required experimental precision on Earth's gravity. It is thus the main objective of the present paper to show that the present theoretical status of the critical point phenomena can predict the GC turbidity data near the xenon liquid-gas critical point without adjustable parameter. The theoretical estimation can also be extended to any other one-component fluid for which the experimental location of its liquid-gas critical point is known in the $p, V, T$ phase surface.

Indeed, it is now well-established (see, for example Ref. [2]). that the asymptotic singularity of the field and density properties of the one-component fluids can be described by the classical-to-critical crossover functions provided by the $\left(\phi^{2}\right)^{2}$ field theory framework (see, for example Ref. [3]). Asymptotically close to the liquid-gas critical point, each onecomponent fluid exhibits the universal features predicted for the complete $\{d=3, n=1\}$ universality class of the three-dimensional (3D), symmetrical, Ising like systems, where $d$ is the space dimension and $n$ is the dimension of the order parameter. Moreover, recent theoretical improvements $[4,5]$ have extended the applicability range of the crossover functions far from the critical point. Nevertheless, their fundamental interest remains their conformity with the accurate Ising-like universal values of the critical (leading and lowest confluent) exponents and (leading and first confluent) amplitude combinations [6], only valid very close to the $\left(\phi^{2}\right)^{2}$ singular point. Consequently, only the crossover description within the so-called Ising-like preasymptotic domain (PAD) [4] can be precisely characterized by a limited number (3) of free, system-dependent, nondimensional parameters. In addition, this description in terms of field and density variables needs to define the energy and length scale units to make any physical properties in a dimensionless form, requiring thus the introduction of the measurable physical critical parameters such as the critical temperature $T_{c}$, the critical pressure $p_{c}$, the critical density $\rho_{c}$, etc. The subscript $c$ refers to a critical quantity. In that dimensionless scheme where the uniqueness of the length unit is essential [7], the Ising-like singular behaviors within the PAD are then characterized by two leading amplitudes and one-confluent amplitude of the two-term Wegner like expansions [8]. This three-parameter characterization results from universal scaling laws and universal amplitude combinations with three independent universal exponents (two leading exponents and the lowest exponent $\Delta$ of the confluent singularities) and, correlatively, the same number (three) of related fluid-dependent amplitudes.

In general, a renormalizable theory cannot predict the location of the critical point or estimate the true extension of the fluid PAD. Therefore, experiments remain the only way to validate the Ising-like behavior and to estimate the limited number of readily independent parameters that (i) localize the critical point and (ii) characterize the crossover phenomena. 
This challenging situation introduces a crucial difficulty in analyzing the experimental data obtained at "finite" distance to the critical point, i.e., in a field (and/or a density) range that can overpass the unknown extension of the PAD. Indeed, beyond the PAD, many other correction terms can also contribute to a Wegner-like expansion at any order beyond the first order term of the confluent corrections to scaling, leading to a non-conclusive Ising-like fluid characterization.

However, the thermodynamic information contained in the experimental location of the isolated liquid-vapor critical point on the experimental phase surface of equation of state $p(T, V, N)=\left(\frac{\partial A}{\partial V}\right)_{T, N}$, permits to overcome the above challenge, as proposed in Refs. [9-11]. The total Helmholtz free energy $A$ is here expressed in terms of its three natural variables, namely, the temperature $T$, the total volume $V$, and the total number of particles $N$. After the normalization of the fluid description per constitutive particle, it is then thermodynamically equivalent to define a minimal number (four) of finite critical coordinates of the critical point location in the particle phase surface of equation of state $p\left(T, v_{\bar{p}}\right)=\left(\frac{\partial a_{\bar{p}}}{\partial v_{\bar{p}}}\right)_{T}$ and to introduce a limited number (five) of asymptotic system-dependent parameters in a renormalizable theory $[12,13] . a_{\bar{p}}=\frac{A(T, V, N)}{N}$ is the Helmholtz free energy of the fluid particle, $v_{\bar{p}}=\frac{V}{N}$ is the volume occupied by the fluid particle and the subscript $\bar{p}$ then refers to a particle quantity. Then, the term-to-term mapping of the physical and theoretical singular behaviors within the PAD completes the critical thermodynamic description by introducing three well-defined "master" (i.e., constant) numbers for all the one-component fluids, which provides the master crossover functions [14], especially for the crossover behaviors of the isothermal compressibility $\kappa_{T}\left(\Delta \tau^{*}, \Delta \widetilde{\rho}=0\right)$ and the coexisting liquid-gas density difference $\Delta \tilde{\rho}_{L V}\left(\left|\Delta \tau^{*}\right|, \Delta \widetilde{\rho}=0\right)$, along the critical isochore. Here $\kappa_{T}=-\frac{1}{V}\left(\frac{\partial V}{\partial p}\right)_{T, N}$, $\Delta \tau^{*}=\frac{T-T_{c}}{T_{c}}, \Delta \tilde{\rho}=\frac{\rho-\rho_{c}}{\rho_{c}}$, and $\Delta \tilde{\rho}_{L V}=\frac{\rho_{L}-\rho_{V}}{2 \rho_{c}}$, where $\rho_{L}$ and $\rho_{V}$ are the coexisting liquid and vapor densities. We are thus able to estimate the PAD extension of any critical fluid, only using its four well-defined critical coordinates. In addition, while the correlation length $\xi$ remains a non-explicit physical parameter in a thermodynamic description, we emphasize that this master description can also provide access to the crossover function $\xi\left(\Delta \tau^{*}, \Delta \widetilde{\rho}=0\right)$. In the above procedure, the xenon behaves as a standard (non-quantum) Ising-like fluid [13,15]. Accordingly, the comparison between the predicted master crossover behaviors (without adjustable parameters) and the experimental measurements of the turbidity of xenon are of basic interest.

Another well-developped approach in representing thermodynamic fluid-properties by a crossover formulation is provided by the crossover parametric model (CPM) [16]. The latter model is issued from a transformed Landau expansion for the Helmholtz energy density, so-called the crossover Landau model (CLM), originally formulated by Chen et al. $[17,18]$. In such a phenomenological crossover approach, Chen et al. have implemented a matchpoint method proposed by Nicoll et al. [19-21]. The resulting crossover formulation also incorporates the accurate universal features of the Ising-like systems close to their critical point. It is then of importance for the renormalization-group methods to provide accurate matching for the Ising-like description within the preasymptotic domain, as well using the above master crossover functions as using the symmetrical CPM functional form. According to the Ising-like similarity, we can also define the crossover master model (CMM) of the one-component-fluid subclass, where the free Ising-like parameters in the CPM model are now calculated from the four well-defined critical coordinates of each pure fluid. Therefore, the comparison with the measured singular behaviors of xenon is important to validate the results predicted from symmetrical CMM. This basic comparison can also provide access to 
the thermodynamic description of the complete phase surface surrounding the vicinity of the liquid-gas critical point of any one-component fluid. If the correlation length is estimated without any supplementary adjustable parameter, we will then open a new route for the complete crossover description of the singular behaviors of the one-component fluid subclass, which will be expected valid for any point in the vicinity of the liquid-vapor critical point of the fluid phase surface.

We shall proceed as follows. In Sect. 2, we provide the main characteristic parameters of the GC experiment and we discuss the conditions to perform theoretical analysis of the turbidity in conformity with the two-scale-factor universality. In Sect. 3, we give the master theoretical estimation of the Ising-like singular behaviors ${ }^{1}$ of $\xi$ and $\kappa_{T}$ only using the four critical coordinates that localize the gas-liquid critical point of xenon. We define precisely the temperature range of the PAD extension where the three-parameter characterization of the fluid is made in accordance with the universal features of the $\{d=3, n=1\}$ universality class. After a brief presentation in Sect. 4 of the fluid turbidity calculated from the OrnsteinZernike (OZ) theory [22,23], we apply in Sect. 5 the Puglielli and Ford functional form [24] to estimate the xenon turbidity. From a detailed analysis of the asymptotic turbidity behavior when $\Delta \tau^{*} \rightarrow 0$, we show that, at large temperature distance from $T_{c}$, the contribution due to the confluent corrections to scaling provides the agreement with the experimental measurements within the claimed experimental uncertainty. We compare also $\xi$ and $\kappa_{T}$ to the previous literature results along the critical isochore. In Sect. 6, we extend the comparison to the results predicted by the crossover master model of the equation of state (EOS), especially for the top-shape of the density coexistence curve, by introducing the master-consistent estimation of the adjustable CPM critical parameters. We conclude in Sect. 7.

\section{Güttinger and Cannell Experiment}

The following analysis is focused on $\xi$ and $\kappa_{T}$ obtained analyzing the optical (diffusion and transmission) responses of a xenon critical cell illuminated by a laser beam. Indeed, the GC experiment provides very accurate measurements of the turbidity, i.e., the light attenuation intensity by transmission through a xenon layer of $19.5 \mathrm{~mm}$ thickness, and the light scattering intensity at $\theta=13^{\circ}$ scattering angle. The reduced temperature range was $9.607 \times 10^{-5} \leq$ $\Delta \tau^{*} \leq 1.001 \times 10^{-1}$ (i.e., $\left.28 \mathrm{mK} \leq T-T_{c} \leq 29 \mathrm{~K}\right)$. The turbidity differences $\tau(T)-\tau\left(T_{\mathrm{or}}\right)$ from a selected turbidity value $\tau\left(T_{\mathrm{or}}\right)$ at a reference temperature $T_{\mathrm{or}}$ are given as a function of $\Delta \tau^{*}$ in Table I of Ref. [1]. This table also reports the data of the relative susceptibility per mass unit $\left(\frac{\chi_{\rho}}{\chi_{\rho}\left(T_{\text {or }}\right)}\right)$ obtained from light scattering intensity measurements. To define $\chi_{\rho}$, we consider a system maintained at constant total volume $V$, characterized by the Helmholtz free energy per volume unit $a\left(T, n_{\bar{p}}\right)=\frac{A(T, V, N)}{V}$. In such a thermodynamic description, the chemical potential per particle $\mu_{\bar{p}}=\left(\frac{\partial a}{\partial n_{\bar{p}}}\right)_{T}$ and the chemical potential per mass unit $\mu_{\rho}=\left(\frac{\partial a}{\partial \rho}\right)_{T}=\frac{\mu_{\bar{p}}}{m_{\bar{p}}}$ are the conjugated variables to the particle number density $n_{\bar{p}}$ and the mass density $\rho=m_{\bar{p}} n_{\bar{p}}$, respectively. $m_{\bar{p}}$ is the particle mass. The subscript $\rho$ now refers to a specific quantity. Therefore, the inverse susceptibility per particle $\left(\chi_{\bar{p}}\right)^{-1}=\left(\frac{\partial \mu_{\bar{p}}}{\partial n_{\bar{p}}}\right)_{T}=$ $\left(\frac{\partial^{2} a}{\partial n_{\bar{p}}^{2}}\right)_{T}$ is associated to the particle number density while the inverse susceptibility per mass

${ }^{1}$ Universal central values of the critical exponents used for $\xi$ and $\kappa_{T}$ in this work are $v=0.6303875$ and $\gamma=1.2395935$ (see Ref. [5]). Accordingly, $\Delta=0.50189$ is the lowest universal value of the confluent exponent. 
unit $\left(\chi_{\rho}\right)^{-1}=\left(\frac{\partial \mu_{\rho}}{\partial \rho}\right)_{T}=\left(\frac{\partial^{2} a}{\partial \rho^{2}}\right)_{T}$ is associated to the mass density, with $\chi_{\rho}=\left(m_{\bar{p}}\right)^{2} \chi_{\bar{p}}$, $\chi_{\bar{p}}=\left(n_{\bar{p}}\right)^{2} \kappa_{T}$ and $\chi_{\rho}=\rho^{2} \kappa_{T}$. Here, $\chi_{\bar{p}}$ (or $\chi_{\rho}$ ) are expected symmetric around the critical density to be compared with the magnetic-like susceptibility function of the $O(1)$ vector of the $\left(\phi^{2}\right)^{2}$ field theory.

In I, the $\chi_{\rho}$ data at $\rho=\rho_{c}$ were obtained by the authors using the Ornstein-Zernike (OZ) theory [22,23] to approximate the light scattering intensity due to the local fluid density fluctuations. It was simultaneously accounted for the singular behavior of the correlation length. Consequently, $\tau\left(\Delta \tau^{*}\right), \xi\left(\Delta \tau^{*}\right)$ and $\kappa_{T}\left(\Delta \tau^{*}\right)=\frac{\chi_{\rho}\left(\Delta \tau^{*}\right)}{\left(\rho_{c}\right)^{2}}$ along the xenon critical isochore were stated to conform to the $\mathrm{OZ}$ theory in spite of its questionable validity when $\Delta \tau^{*} \rightarrow 0$. Moreover, it is also noticeable that the universal features of the Ising-like systems are not completely accounted for in their $\xi\left(\Delta \tau^{*}\right)$ and $\kappa_{T}\left(\Delta \tau^{*}\right)$ fitting functions, especially at the first order of the confluent corrections-to-scaling.

Alternatively, in the present approach, all these Ising-like universal features can be precisely controlled without any adjustable parameter through the non-dimensional master crossover functions $\xi\left(\Delta \tau^{*}\right)$ and $\kappa_{T}\left(\Delta \tau^{*}\right)$ given in Ref. [14] (see also Eqs. (1) and (2) below). Therefore, $\xi\left(\Delta \tau^{*}\right)$ and $\kappa_{T}\left(\Delta \tau^{*}\right)$, (or $\chi_{\rho}\left(\Delta \tau^{*}\right)$ and $\chi_{\bar{p}}\left(\Delta \tau^{*}\right)$ ), being known a priori, our following calculation of $\tau\left(\Delta \tau^{*}\right)$ is only depending on the theoretical functional form that connects $\xi$ and $\kappa_{T}$ (or $\chi_{\rho}, \chi_{\bar{p}}$ ) to the light scattering intensity $I(\theta)$ as a function of the scattering angle $\theta$.

In a general fluid theory assuming spatial isotropy, the magnitude and the spatial character of the local density fluctuations are described in terms of the pair correlation function $g(r)$ or, equivalently, in terms of the static structure factor $S(q)$, where $q$ is the wavenumber amplitude of the momentum transfer wave vector. $S(q)$ is proportional to the intensity $I(q)$ of the scattered electromagnetic radiation by the transparent fluid $f$ of refractive index $n_{f}$, for the Bragg condition $q=\frac{4 \pi n_{f}}{\lambda} \sin \frac{\theta}{2}$ at (generally small) angle $\theta$ (see, for example Ref. [25]). The $q$-scattering experiments provide thus a distinction between light scattering experiments (typical wavelength range $\lambda \sim 500-700 \mathrm{~nm}$ ) and X-ray or neutron scattering experiments (typical wavelength range $\lambda \sim 0.4-1 \mathrm{~nm}$ ). Accordingly, $S(q)$ is a measurable quantity for any local fluid theory able to calculate the thermodynamic equilibrium from the representation of the fluid microscopic structure by the radial distribution of the particles. We recall that $S(0)$ is a purely thermodynamic quantity proportional to the scattered intensity $I(0)$ at zeroangle $(q=0)$. In a one-component fluid description per particle, $S(0)=n_{\bar{p}} k_{B} T \chi_{\bar{p}}$, since the zeroth-moment of the pair correlation function is related to $\chi_{\bar{p}}$, due to the fluctuationdissipation theorem.

In a critical fluid theory, the normalization of $\frac{S(q)}{k_{B} T}$ appears essential in order to correctly account for the physical dimension of the selected symmetrized susceptibility ${ }^{2}$ in pure fluids (see Ref. [26]). As previously mentioned, when the susceptibility $\chi_{\bar{p}}$ (or $\chi_{\rho}$ ) appears symmetric around the zero value of the related order parameter [27], the singular behaviors of a one-component fluid very close to the liquid-gas critical point must behave similarly to the ones of the $O(1)$ vector model of the symmetric $\left(\phi^{2}\right)^{2}$ field theory and the simple cubic scalar $(N=1)$ lattice model of 3D-Ising systems. In such an ideal Ising-like asymptotic description which complies with the two-scale factor universality when $T \rightarrow T_{c}$, the expected behaviors of the static structure factor [28-33], and the turbidity [32,33], must involve only three asymptotic amplitude-exponent pairs (neglecting thus the contribution of the confluent sin-

\footnotetext{
2 A theoretical description of $I\left[q, \xi\left(\Delta \tau^{*}, \Delta n_{\bar{p}}^{*}\right)\right]$ needs to generally assume symmetrized singular behaviors of the isothermal susceptibility and the correlation length around the critical isochore $n_{\bar{p}}=n_{\bar{p}}, c$.
} 
gularities in the Wegner-like expansions). These three asymptotic amplitude-exponent pairs are:

i) $\left\{\Gamma_{0}^{+}, \gamma\right\}$ pair of the singular pure power law $\kappa_{T}=\Gamma_{0}^{+}\left(\Delta \tau^{*}\right)^{-\gamma}$ describing the asymptotic temperature dependence of the physical isothermal compressibility,

ii) $\left\{\xi_{0}^{+}, v\right\}$ pair of the singular pure power law $\xi=\xi_{0}^{+}\left(\Delta \tau^{*}\right)^{-v}$ describing the asymptotic temperature dependence of the physical correlation length, and

iii) $\left\{C_{\infty}^{+}, \eta\right\}$ universal pair describing the Ising-like asymptotic dependence $G(x)=\frac{C_{\infty}^{+}}{x^{2-\eta}}$ of the universal scaling function for $x \rightarrow \infty . G(x)=\frac{S\left[q, \Delta \tau^{*}, \xi\left(\Delta \tau^{*}\right)\right]}{S\left[0, \Delta \tau^{*}, \xi\left(\Delta \tau^{*}\right)\right]}$ only depends on the single variable $x=q \xi$ ( $x$ is non-dependent of the normalization scheme of the physical quantities), while $\eta$ is the so-called Green-Fisher critical exponent [28,29].

In that asymptotic Ising limit, $\Gamma_{0}^{+}$and $\xi_{0}^{+}$are used as the fluid-dependent parameters. The two-scale factor universality is accounted for by the universal scaling law $\gamma=v(2-\eta)$ and the universal amplitude combination $Q_{3}=\frac{\hat{D}_{\rho, 0}\left(\xi_{0}^{+}\right)^{2-\eta}}{\rho_{c}^{2} \Gamma_{0}^{+}} \equiv C_{\infty}^{+}=\frac{\chi(q) \xi^{2-\eta}}{\chi(0)}=G(x) x^{2-\eta}$ [29-31,33], where:

i) $\gamma$ and $v$ are the two leading critical exponents,

ii) $\Gamma_{0}^{+}$and $\xi_{0}^{+}$are the two entry physical amplitudes,

iii) $\left\{\hat{D}_{\rho, 0}, \eta\right\}$ is the resulting (physical) amplitude-(universal) exponent pair in the thermodynamic description per mass unit.

The critical point condition $\xi \sim \infty$ at $\Delta \tau^{*}=0$ and $\Delta \rho^{*}=0$ is here labelled by the hat decoration. Therefore, in momentum space at the critical point, the fluid-dependent amplitude $\hat{D}_{\rho, 0}$, of $\chi_{\rho}(q, \xi=\infty)=\frac{\hat{D}_{\rho, 0}}{q^{2-\eta}}$ can be estimated unambiguously from $\gamma, v, \Gamma_{0}^{+}$and $\xi_{0}^{+}$.

Moreover, as $\xi$ and $\kappa_{T}$ are hereafter predicted without any adjustable parameter, we are also able to verify that the temperature range $\Delta \tau^{*} \geq 9.6 \times 10^{-5}\left(T-T_{c} \geq 28 \mathrm{mK}\right)$ covered by GC experiments is well beyond the upper PAD limit $\mathcal{L}_{\mathrm{PAD}}^{\mathrm{Xe}} \simeq 9 \times 10^{-5}\left(T-T_{c} \leq 26 \mathrm{mK}\right.$, see below). It is also easy to show that the light scattering experiments at $\theta=13^{\circ}$, i.e., $q=2.23 \times 10^{6} \mathrm{~m}^{-1}$, with $\lambda_{0}=632.8 \mathrm{~nm}$ and critical refractive index of xenon $n_{\mathrm{Xe}, c}=$ 1.1375 [34,35], are covering the range $2.0 \times 10^{-3} \leq q \xi \leq 0.14$ (see below). The condition $q \xi<1$ being satisfied, the truncated Taylor serie expansion of the universal scaling function is expected similar to the $\mathrm{OZ}$ approximant $[G(x)]^{-1}=1+x^{2}$. In such a situation, GC measurements can then be analyzed introducing the complete Wegner expansion given by a classical-to-critical crossover theory. As a final consequence, GC turbidity measurements can demonstrate unambiguously that the deviation from the Ising-like turbidity is entirely accounted for by the contribution of the confluent corrections to scaling.

\section{Ising-Like Singular Behaviors of $\xi$ and $\kappa_{T}$ for Xenon Case}

The estimation of $\xi$ and $\kappa_{T}$ following the method proposed in Ref. [14] only needs to know 4 critical parameters of xenon, namely, the critical temperature $T_{c}$, the critical pressure $p_{c}$, the critical mass density $\rho_{c}$, and the common critical slope $\gamma_{c}^{\prime}=\left[\left(\frac{\partial p}{\partial T}\right)_{\rho=\rho_{c}}=\frac{d p_{\text {sat }}}{d T}\right]_{T \rightarrow T_{c}^{ \pm}}$ of the critical isochore $\left(T \rightarrow T_{c}^{+}\right)$and the saturation pressure $p_{\text {sat }}(T)$ curve $\left(T \rightarrow T_{c}^{-}\right)$. Their values, given in Table 1 (see Refs. $[15,36]$ ), localize the vapor-liquid critical point of xenon on the $p, v_{\bar{p}}, T$ phase surface. $v_{\bar{p}}=\frac{m_{\bar{p}}}{\rho} . M_{\text {mol }}=N_{A} m_{\bar{p}}$ is the molar mass of the fluid. $N_{A}$ is the Avogadro's number with $R=N_{A} k_{B} . R$ is the ideal gas constant and $k_{B}$ is 
Table 1 Critical coordinates and physical quantities for xenon (with

$M_{\mathrm{mol}}(\mathrm{Xe})=131.29 \mathrm{~g} \mathrm{~mol}^{-1}$ and $N_{A}=$

$6.02214129 \times 10^{23} \mathrm{~mol}^{-1}$ ), and characteristic parameters for the light transmission experiments of GC (see also Refs. given in the last column)

\begin{tabular}{|c|c|c|}
\hline & $\mathrm{Xe}$ & Ref. \\
\hline$m_{\bar{p}}$ & $2.1805 \times 10^{-25} \mathrm{~kg}$ & \\
\hline$T_{c}$ & $289.733 \pm 0.015 \mathrm{~K}$ & {$[15,36]$} \\
\hline$p_{c}$ & $5.84007 \pm 0.00050 \mathrm{MPa}$ & [15] \\
\hline$\rho_{c}$ & $1113 \pm 3 \mathrm{~kg} \mathrm{~m}^{-3}$ & “ \\
\hline$\gamma_{c}^{\prime}$ & $0.1197 \pm 0.0006 \mathrm{MPa} \mathrm{K}^{-1}$ & “ \\
\hline$\left(\beta_{c}\right)^{-1}=k_{B} T_{c}$ & $4.0002 \times 10^{-21} \mathrm{~J}$ & “ \\
\hline$\alpha_{c}=\left(\frac{k_{B} T_{C}}{p_{c}}\right)^{\frac{1}{d}}$ & $8.81498 \times 10^{-10} \mathrm{~m}$ & “ \\
\hline$Y_{c}=\gamma_{c}^{\prime} \frac{T_{c}}{p_{c}}-1$ & 4.93846 & “ \\
\hline$Z_{c}=\frac{p_{c} m_{\bar{p}}}{\rho_{c} k_{B} T_{c}}$ & 0.28602 & “ \\
\hline$n_{\mathrm{Xe}, c}$ & 1.1375 & {$[34,35]$} \\
\hline$L L_{c}$ & $10.5271 \mathrm{~cm}^{3} \mathrm{~mole}^{-1}$ & “ \\
\hline$A_{0} k_{B}$ & $(88.49 \pm 0.30) \mathrm{J} \mathrm{K}^{-1} \mathrm{~m}^{-4}$ & “ \\
\hline$T_{\mathrm{or}}-T_{c}$ & $0.6677 \mathrm{~K}$ & {$[1]$} \\
\hline$\chi_{\rho}\left(T_{\mathrm{or}}\right)$ & $2.42 \times 10^{-7} \mathrm{~kg}^{2} \mathrm{~J}^{-1} \mathrm{~m}^{-3}$ & " \\
\hline$k_{0}$ & $1.12944 \times 10^{7} \mathrm{~m}^{-1}$ & “ \\
\hline$\tau\left(T_{\mathrm{or}}\right)$ & $4.1 \mathrm{~m}^{-1}$ & “ \\
\hline$\tau_{0}$ & $7.975 \times 10^{-4} \mathrm{~m}^{-1}$ & “ \\
\hline
\end{tabular}

the Boltzmann's constant. From these critical coordinates we can calculate 4 unequivocal values of the:

(i) energy unit $\left(\beta_{c}\right)^{-1}=k_{B} T_{c}$;

(ii) length unit $\alpha_{c}=\left(\frac{k_{B} T_{c}}{p_{c}}\right)^{\frac{1}{d}}$ with $d=3$, where $\left(\alpha_{c}\right)^{d}=\frac{k_{B} T_{c}}{p_{c}}$ is the characteristic critical volume of the molecular interaction cell [9-11];

(iii) first scale factor $Y_{c}=\gamma_{c}^{\prime} \frac{T_{c}}{p_{c}}-1$ of the dimensionless thermal field $\Delta \tau^{*}=\frac{T-T_{c}}{T_{c}}$;

(iv) second scale factor $Z_{c}=\frac{p_{c} m_{\bar{p}}}{\rho_{c} k_{B} T_{c}}$ of the dimensionless ordering field $\Delta \mu_{\bar{p}}^{*}=$ $\left(\mu_{\bar{p}}-\mu_{\bar{p}, c}\right) \beta_{c}$ conjugated to the dimensionless order parameter density $\Delta n_{\bar{p}}^{*}=$ $\left(n_{\bar{p}}-n_{\bar{p}, c}\right)\left(\alpha_{c}\right)^{d} .\left(Z_{c}\right)^{-1}=n_{\bar{p}, c}\left(\alpha_{c}\right)^{d}$ is the characteristic critical number of particles filling the molecular interaction cell [9-11].

We recall that $\Delta \mu_{\bar{p}}^{*}=Z_{c} \Delta \tilde{\mu}_{\rho}$ and $\Delta n_{\bar{p}}^{*}=\frac{\Delta \widetilde{\rho}}{Z_{c}}$, where $\Delta \tilde{\mu}_{\rho}=\left(\mu_{\rho}-\mu_{\rho, c}\right) \frac{\rho_{c}}{p_{c}}$ and $\Delta \widetilde{\rho}=$ $\frac{\rho-\rho_{c}}{\rho_{c}}$ are the practical conjugated ordering field and order parameter density, respectively (for a fluid of mass unit). Accounting now for the extensive nature of the fluid susceptibility as already made in Refs. [14,15], the dimensionless susceptibilities $\tilde{\chi}=\left(\frac{\partial \Delta \widetilde{\rho}}{\partial \Delta \widetilde{\mu}_{\rho}}\right)_{\Delta \tau^{*}}=$ $\chi_{\rho} \frac{p_{c}}{\left(\rho_{c}\right)^{2}} \equiv \tilde{\chi}_{\rho}$ (for a fluid of mass unit), and $\chi^{*}=\left(\frac{\partial \Delta n_{\bar{p}}^{*}}{\partial \Delta \mu_{\bar{p}}^{*}}\right)_{\Delta \tau^{*}}=\chi_{\bar{p}} \frac{\left(\alpha_{c}\right)^{d}}{\beta_{c}} \equiv \chi_{\bar{p}}^{*}$ (for a fluid particle), with $\tilde{\chi}_{\rho}=\left(Z_{c}\right)^{2} \chi_{\bar{p}}^{*}$, are related to the dimensionless order parameter densities $\Delta \tilde{\rho}$ and $\Delta n_{\bar{p}}^{*}$, respectively. We underline here the importance of the length and energy normalizations for any thermodynamic description, which can then introduce the non-dimensional scale factor $Z_{c}$ (in analytic powered forms) when another length unit or energy unit is used in addition to $\alpha_{c}$ and $\left(\beta_{c}\right)^{-1}$. That is precisely the case for a quantity 
decorated by a tilde, in spite of a similar non-dimensional potential density, since $\widetilde{a}_{\rho}=\frac{a}{p_{c}}=$ $a \beta_{c}\left(\alpha_{c}\right)^{d}=a^{*}$.

Neglecting quantum effects [37] in xenon case, $\xi_{\text {cal }}^{*}\left(\Delta \tau^{*}\right)=\left(\frac{1}{\alpha_{c}}\right) \times \xi\left(\Delta \tau^{*}\right)$ and $\kappa_{T, \text { cal }}^{*}\left(\Delta \tau^{*}\right)=p_{c} \times \kappa_{T, \text { cal }}\left(\Delta \tau^{*}\right)$ are thus computed from the following equations

$$
\begin{aligned}
\xi_{\text {cal }}^{*}\left(\Delta \tau^{*}\right) & =\ell^{*}\left(\mathcal{T}^{*}\right)=\frac{1}{\mathbb{Z}_{\xi}^{\{1 f\}}\left[\ell_{t h}(t)\right]^{-1}} \\
Z_{c} \times \kappa_{T, \text { cal }}^{*}\left(\Delta \tau^{*}\right) & =\mathcal{X}^{*}\left(\mathcal{T}^{*}\right)=\frac{1}{\mathbb{Z}_{\chi}^{\{1 f\}}\left[\chi_{t h}(t)\right]^{-1}}
\end{aligned}
$$

with $t=\Theta^{\{1 f\}} \mathcal{T}^{*}=\Theta^{\{1 f\}} Y_{c} \Delta \tau^{*}=\vartheta \Delta \tau^{*}$, i.e., $\mathcal{T}^{*}=Y_{c} \Delta \tau^{*}$ and $\vartheta=\Theta^{\{1 f\}} Y_{c}$. The superscript $\{1 f\}$ recalls for a constant quantity which characterizes the one-component fluid subclass. In Eqs. (1) and (2), the master crossover functions $\ell^{*}\left(\mathcal{T}^{*}\right)$ and $\mathcal{X}^{*}\left(\mathcal{T}^{*}\right)$ [14] correspond to the modifications of the theoretical crossover functions $\left[\ell_{t h}(t)\right]^{-1}$ and $\left[\chi_{t h}(t)\right]^{-1}$ defined in Refs. [4,5]. These modifications only introduce three scale factors $\Theta^{\{1 f\}}=4.28810^{-3}, \Psi^{\{1 f\}}=1.7410^{-4}$ and $\mathbb{L}_{f}=25.6988$, to characterize the onecomponent fluid subclass [14]. Accordingly, the master values of the prefactors appearing in Eqs. (1) and (2) are $\mathbb{Z}_{\xi}^{\{1 f\}} \equiv \mathbb{L}_{f}=25.6988$ and $\mathbb{Z}_{\chi}^{\{1 f\}}=\left(\mathbb{L}_{f}\right)^{d}\left(\Psi^{\{1 f\}}\right)^{2}=1950.70$, while the ones of the theoretical and master leading amplitudes of the related crossover functions are $\left(\mathbb{Z}_{\xi}^{+}\right)^{-1}=0.471474, \mathcal{Z}_{\xi}^{+}=0.5729,\left(\mathbb{Z}_{\chi}^{+}\right)^{-1}=0.269571$ and $\mathcal{Z}_{\chi}^{+}=0.11975$, with $\mathcal{Z}_{\xi}^{+} \mathbb{Z}_{\xi}^{+}=\left[\mathbb{L}_{f}\left(\Theta^{\{1 f\}}\right)^{\nu}\right]^{-1}$ and $\mathcal{Z}_{\chi}^{+} \mathbb{Z}_{\chi}^{+}=\left[\left(\mathbb{L}_{f}\right)^{d}\left(\Psi^{\{1 f\}}\right)^{2}\left(\Theta^{\{1 f\}}\right)^{\gamma}\right]^{-1}$ (the subscript + refers to the homogeneous domain $T>T_{c}$ ).

Equations 1 and 2 can be written in their usual forms $\xi=\xi_{0}^{+}\left(\Delta \tau^{*}\right)^{-v} L_{\Delta}\left(\Delta \tau^{*}\right)$ and $\kappa_{T}=\Gamma_{0}^{+}\left(\Delta \tau^{*}\right)^{-\gamma} K_{\Delta}\left(\Delta \tau^{*}\right)$ where the asymptotic Ising power laws are corrected by the confluent crossover functions $L_{\Delta}\left(\Delta \tau^{*}\right)$ and $K_{\Delta}\left(\Delta \tau^{*}\right)$. The subscript refers to the lowest critical exponent $\Delta=0.50189[4,5]$. Indeed, $L_{\Delta}\left(\Delta \tau^{*}\right)=\left[1+\sum_{i=1}^{\infty} a_{\xi}^{i,+}\left(\Delta \tau^{*}\right)^{i \Delta}\right]$ and $K_{\Delta}\left(\Delta \tau^{*}\right)=\left[1+\sum_{i=1}^{\infty} a_{\chi}^{i,+}\left(\Delta \tau^{*}\right)^{i \Delta}\right]$ are the resummations of the complete Wegner-like expansions, so-called the $\Delta$-contribution in the following (see details in Refs. $[4,5]$ ). The confluent singularities of the classical-to-critical crossover are only due to $\Delta$, with the universal ratio $\frac{a_{\xi}^{1,+}}{a_{\chi}^{1,+}}=\frac{\mathbb{Z}_{\xi}^{1,+}}{\mathbb{Z}_{\chi}^{1,+}}=\frac{\mathcal{Z}_{\xi}^{1,+}}{\mathcal{Z}_{\chi}^{1,+}}=0.68$. This universal ratio means that only $a_{\chi}^{1,+}\left(\right.$ or $\left.a_{\xi}^{1,+}\right)$ is the first-order amplitude characteristic of the fluid, which acts as a single crossover parameter. $\xi$ and $\kappa_{T}$ singular behaviors for xenon are then predicted from Eq. (1) and Eq. (2) to be in accordance with the universal features of the three-dimensional Ising-like universality class at the first-order of the confluent corrections (see Table 2).

Accordingly, $\xi=\alpha_{c} \xi^{+}\left(\Delta \tau^{*}\right)^{-v}$ with $\xi_{0}^{+}=\alpha_{c} \xi^{+}=\alpha_{c}\left(Y_{c}\right)^{-v} \mathcal{Z}_{\xi}^{+}=0.184531 \mathrm{~nm}$, (i.e., $\left.\xi^{+}=0.209338\right)$ and $p_{c} \kappa_{T}=\Gamma^{+}\left(\Delta \tau^{*}\right)^{-\gamma}$ with $\Gamma^{+}=\left(Z_{c}\right)^{-1}\left(Y_{c}\right)^{-\gamma} \mathcal{Z}_{\chi}^{+}=$ 0.0578238 , (i.e., $\Gamma_{0}^{+}=9.901 \mathrm{MPa}^{-1}$ ) (using standard notations and values of Ref. [5]). The non-dimensional leading amplitude $\hat{D}_{\rho}=\frac{\hat{\mathcal{Z}}_{G}}{Z_{c}}$ satisfies the universal amplitude combination $Q_{3} \equiv C_{\infty}^{+}=\frac{\hat{D}_{\rho}\left(\xi^{+}\right)^{2-\eta}}{\Gamma^{+}} \simeq 0.92$ [30,31]. Similarly, the non-dimensional leading amplitude $B=\left(Z_{c}\right)^{-\frac{1}{2}}\left(Y_{c}\right)^{\beta} \mathcal{Z}_{M}=1.46762$ involved in the power law $\Delta \widetilde{\rho}_{L V}=B\left(\Delta \tau^{*}\right)^{\beta}$ satisfies the universal amplitude combination $R_{C}^{+}\left(R_{\xi}^{+}\right)^{-d}=\frac{\Gamma^{+}}{B^{2}}\left(\xi^{+}\right)^{-d}=2.92922$. There- 
Table 2 Estimated universal features in the master form (column 2) of the MR scheme and in the CPM (column 3)

\begin{tabular}{|c|c|c|c|}
\hline & MR [4] & CPM [16] & $R \%$ \\
\hline \multicolumn{4}{|l|}{ Exponents } \\
\hline$\gamma$ & 1.2395935 & 1.239 & -0.048 \\
\hline$\beta$ & 0.3257845 & 0.3255 & -0.087 \\
\hline$\alpha$ & 0.1088375 & 0.110 & 1.07 \\
\hline$v$ & 0.6303875 & 0.630 & -0.061 \\
\hline$\eta$ & 0.033604 & 0.0333 & -0.90 \\
\hline$\Delta$ & 0.50189 & 0.52 & 3.6 \\
\hline \multicolumn{4}{|l|}{ Combinations } \\
\hline$R_{C}^{+}=\frac{\Gamma^{+}}{B^{2}} A^{+}$ & $0.0574 \pm 0.0020$ & 0.0580 & 1.05 \\
\hline$R_{\xi}^{+}=A^{+}\left(\xi^{+}\right)^{\frac{1}{d}}$ & $0.2696 \pm 0.0007$ & 0.2659 & -1.37 \\
\hline$R_{C}^{+}\left(R_{\xi}^{+}\right)^{-d}=\frac{\Gamma^{+}}{B^{2}}\left(\xi^{+}\right)^{-d}$ & $(2.92922)$ & $(3.0851)$ & 5.32 \\
\hline$\frac{\xi^{+}}{\xi^{-}}$ & $(1.96)$ & $(1.96)$ & $(0)$ \\
\hline$\frac{\Gamma^{+}}{\Gamma^{-}}$ & $4.79 \pm 0.010$ & 4.94 & 3.13 \\
\hline$\frac{A_{\alpha}^{+}}{A_{\alpha}^{-}}=\frac{A^{+}}{A^{-}}$ & $0.537 \pm 0.019$ & 0.524 & -2.42 \\
\hline$\frac{a_{\xi}^{+}}{a_{\chi}^{+}}$ & $0.68 \pm 0.02$ & 0.44 & -35.3 \\
\hline$\frac{a_{\mathbb{C}}^{+}}{a_{\chi}^{+}}=\frac{A_{1}^{+}}{\alpha a_{\chi}^{+}}$ & $8.68 \pm 0.23$ & 6.872 & -20.7 \\
\hline$\frac{a_{\mathbb{M}}}{a_{\chi}^{+}}$ & $0.90 \pm_{0.85}^{0.21}$ & 0.897 & 0.33 \\
\hline$\frac{a_{\xi}^{+}}{a_{\xi}^{-}}$ & (1.1) & & \\
\hline$\frac{a_{x}^{+}}{a_{\chi}^{-}}$ & $0.215 \pm 0.029$ & 0.195 & -9.3 \\
\hline$\frac{a_{\mathbb{C}}^{+}}{a_{\mathbb{C}}^{-}}$ & $1.20 \pm_{0.31}^{0.63}$ & 0.827 & -31.1 \\
\hline
\end{tabular}

Residuals $R \%=100 \times\left(\frac{C P M}{M R}-1\right)($ column 4$)$

fore, considering the amplitude set $\xi^{+}, \hat{D}_{\rho}, \Gamma^{+}$and $B$, any dimensionless amplitude value ( $\xi^{+}$and $\hat{D}_{\rho}$ here above) can be estimated when two of them are known and selected as independent ( $\Gamma^{+}$and $B$ in Ref. [15]), in agreement with the two-scale factor universality.

Relaying the GC conclusive sentence in I, the main interest of Eqs. (1) and (2) remains the estimation of the confluent corrections-to-scaling at finite distance to $T_{c}$, only using a single crossover parameter associated to $\Delta$. In addition, the $\Delta$-contribution can be computed for the complete classical-to-critical crossover domain without any adjustable parameter, selecting for example $a_{\chi}^{1,+}$ as the independent first-order amplitude, which acts as a fluid crossover parameter. Since the critical density of the xenon cell was well-controled at the level of the illuminated volume under the field acceleration due to Earth's gravity, Eqs. (1) and (2) can be directly used to predict the GC results along the critical isochore and then to quantify the $\Delta$-contribution to $\xi, \kappa_{T}$, and $\tau$ (see below Sect. 4), in the covered experimental range. 


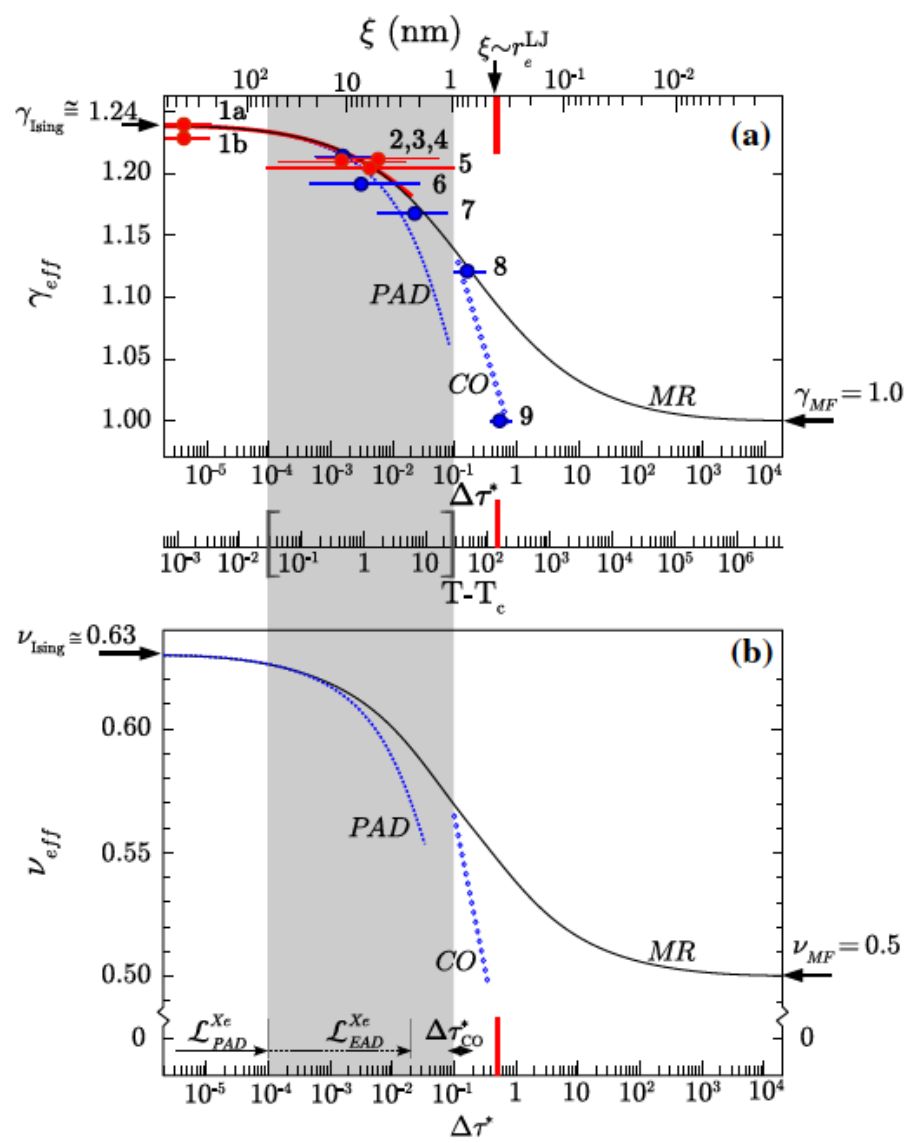

Fig. 1 Log-linear scale: MR (black) continuous curves Computed crossover behavior of $\gamma_{e}$ (a) and $v_{e}$, (b) from Eqs. (2) and (1) respectively, as functions of $\Delta \tau^{*}, T-T_{c}(\mathrm{~K})$, or $\xi(\mathrm{nm})$ (lower, medium, and upper horizontal axes, respectively). PAD (blue) doted curves ibid from Eqs. (12) and (11) respectively. $\gamma_{e}($ red) curve in (a) computed from a GC fit (see text). Horizontal(red and blue) segments with (full) circle (IF+LS and $p V T$ ) $\gamma_{e}$-values published before I (see text and Appendix with numbered labels and ranges in Table 6); CO (blue) circle curves abrupt physical crossover (for guidance only). Horizontal exponent arrows (subscripts Ising and MF): Ising $\left(\Delta \tau^{*} \rightarrow 0\right)$ and mean field $\left(\Delta \tau^{*} \rightarrow \infty\right)$ values. Horizontal arrows (lower $\Delta \tau^{*}$ axe): PAD and EAD extensions for xenon (see text). Horizontal double arrow (lower $\Delta \tau^{*}$ axe): restricted temperature range $\Delta \tau_{\mathrm{CO}}^{*}$ of the physical crossover. Grey area GC experimental range bracketed on the $T-T_{c}$ axis. Vertical (red) segments $\xi=r_{e}^{\mathrm{LJ}}=2^{\frac{1}{6}} \sigma^{\mathrm{LJ}}$ with $\sigma^{\mathrm{LJ}} \simeq 0.36 \mathrm{~nm}$ (see text) (Color figure online)

For the $\xi$ and $\kappa_{T}$ cases, the $\Delta$-contributions are illustrated in Fig. 1a, b by following the Kouvel and Fisher approach [38], which introduces the crossover behavior of the effective exponents $\gamma_{e}=-\left(\frac{d \ln \kappa_{T}}{d \ln \Delta \tau^{*}}\right)$ and $v_{e}=-\left(\frac{d \ln \xi}{d \ln \Delta \tau^{*}}\right)$ at finite temperature distances to $T_{c}$. The (lower, medium, and upper) horizontal logarithmic axes of Fig. 1 are labelled in terms of $\Delta \tau^{*}, T-T_{c}(\mathrm{~K})$, and $\xi(\mathrm{nm})$, respectively. The $\xi-\Delta \tau^{*}$ correspondence was computed from Eq. (1). For the complete crossover, the MR black curves show the continuous $\gamma_{e}$ - and $v_{e}$-decrease from the Ising limiting values $\gamma \equiv \gamma_{\text {Ising }} \simeq 1.24$ and $v \equiv v_{\text {Ising }} \simeq 0.63$ when $\Delta \tau^{*} \rightarrow 0$ to the mean-field limiting values $\gamma_{\mathrm{MF}}=1$ and $\nu_{\mathrm{MF}}=\frac{1}{2}$ when $\Delta \tau^{*} \rightarrow \infty$ (see also Ref. [5]). The red $\gamma_{e}$-curve (part (a) of the figure), which matches $( \pm 1 \%)$ the black $\gamma_{e}$-curve 
in the range $\Delta \tau^{*} \leq 5 \times 10^{-2}$, corresponds to $\gamma_{e}$ computed from the GC fit

$$
\kappa_{T}=0.0577\left(\Delta \tau^{*}\right)^{-1.241}\left[1+1.29\left(\Delta \tau^{*}\right)^{0.496}-1.55\left(\Delta \tau^{*}\right)^{0.992}+1.9\left(\Delta \tau^{*}\right)^{1.488}\right]
$$

reported in I. The PAD curves show the exponent decrease only due to the first-order term of the confluent singularities, strictly valid within the preasymptotic domain such as $\Delta \tau^{*} \leq$ $\mathcal{L}_{\mathrm{PAD}}^{\mathrm{Xe}}=\frac{\mathcal{L}_{\mathrm{PAD}}^{\{1 f\}}}{Y_{c}(\mathrm{Xe})} \simeq 9 \times 10^{-5}$ (see the related arrow in lower axis and Ref. [14] for the estimation of $\left.\mathcal{L}_{\mathrm{PAD}}^{\{1 f\}} \simeq 4.43 \times 10^{-4}\right)$. The grey area, bracketed on the $T-T_{c}(\mathrm{~K})$ axis, corresponds to the $\mathrm{GC}$ temperature range. The expected $\xi$-variation in I covers the range $63 \mathrm{~nm} \geq \xi \geq 0.9 \mathrm{~nm}$ while the $\gamma_{e}$ and $v_{e}$-variations remain such as $\gamma<\gamma_{e}<\frac{\gamma+\gamma_{\mathrm{MF}}}{2} \simeq 1.12$ and $v<v_{e}<$ $\frac{v+\nu_{\mathrm{MF}}}{2} \simeq 0.565$. That includes the extended asymptotic domain $\Delta \tau^{*} \leq \mathcal{L}_{\mathrm{EAD}}^{\mathrm{Xe}} \simeq 2 \times 10^{-2}$ (see corresponding arrow in lower axis), which was already the object of a detailed analysis in Refs. [15,40]. We have also reported in Fig. 1a the $\gamma_{e}$-values published from fitting $\kappa_{T}^{*}$ data obtained before GC work using an effective power law $\kappa_{T}^{*}=\Gamma_{e}^{+}\left(\Delta \tau^{*}\right)^{-\gamma_{e}}$ over a finite, restricted temperature range $0<\Delta \tau_{\min }^{*} \leq \Delta \tau^{*} \leq \Delta \tau_{\max }^{*}$ (see Appendix for the data sources). In Fig. 1a, each $\gamma_{e}$ value is shown as an horizontal i-segment of extension $\Delta \tau_{\min }^{*}-\Delta \tau_{\max }^{*}$ (with label i related to line \#i of Table 6). The position of the (full) circle in the segment corresponds to the geometrical mean value $\left\langle\Delta \tau_{\exp }^{*}\right\rangle=\left(\Delta \tau_{\min }^{*} \Delta \tau_{\max }^{*}\right)^{\frac{1}{2}}$. In the Ising-like side of the crossover where $\gamma \leq \gamma_{e}<\gamma_{\frac{1}{2}}$ (left part of Fig. 1a), the circle follows the MR curve in a very satisfactory manner when $\left\langle\Delta \tau_{\exp }^{*}\right\rangle$ changes, at least for the extended asymptotic domain $\Delta \tau^{*} \leq \mathcal{L}_{\mathrm{EAD}}^{\mathrm{Xe}} \simeq 2 \times 10^{-2}$ where the Ising-like nature of the crossover is prominent.

The left half part of Fig. 1a illustrates the experimental status at the mid-80's when was performed the first crossover analysis [13] of xenon singular properties with only 3 adjustable physical parameters, using the crossover functions [13] estimated from the MR scheme (see also below line $\sharp 5$ of Table 4 ). In such initial situation refering to a single characteristic scale factor $\vartheta$ associated to the critical isochoric line of xenon, the crossover behavior of any effective exponent appeared certainly governed by the Ising-like nature of the singularity due to the lowest confluent correction-to-scaling (anticipating then posterior analyses, see lines $\sharp 6$ to $\sharp 8$ of Table 4 , which have lead to similar conclusions independently of the theoretical scheme at the origin of the crossover functions). Today, the master crossover prediction of the Ising-like critical scaling for $\gamma_{e}\left(\Delta \tau^{*}\right)$ is made without adjustable parameter and complies with the universal features of the MR crossover functions of Ref. [4].

However, the dotted lines labeled CO of Fig. 1 interpolating the $\frac{\gamma+\gamma_{\mathrm{MF}}}{2}$ (or $\frac{v+v_{\mathrm{MF}}}{2}$ ) and $\gamma_{\mathrm{MF}}\left(\right.$ or $v_{\mathrm{MF}}$ ) values, are also indicative of the abrupt experimental crossover, generally observed in pure fluids far from the critical point. The related double arrow labeled $\Delta \tau_{\mathrm{CO}}^{*}$ in Fig. 1b, differenciates the high temperature range (right half part of Fig. 1) where a mean-field approximation of a fluid theory is probably questionable. Indeed, the vertical line labelled $r_{e}^{\mathrm{LJ}}$ indicates the temperature distance where the calculated correlation length behaves similar to the equilibrium position $r_{e}^{\mathrm{LJ}}=0.4 \mathrm{~nm} \approx \frac{\alpha_{c}}{2}$ between a pair of xenon atoms interacting from a 12-6 Lennard-Jones form of the pairwise additive potential (with $r_{e}^{\mathrm{LJ}}=2^{\frac{1}{6}} \sigma^{\mathrm{LJ}}$ and $\left.\sigma^{\mathrm{LJ}}=0.3607 \mathrm{~nm}[39]\right)$. The related temperature $T \simeq 550 \mathrm{~K}$ corresponds to a reduced temperature $T^{*}=\frac{k_{B} T}{\epsilon^{\mathrm{LJ}}} \approx 1.9$ (i.e., $\Delta \tau^{*} \approx 0.9$ ). However, for $T^{*} \geq 2$, it is well-known that treating the whole Lennard-Jones potential as a small perturbation of a hard sphere appears as a more realistic approximation than the mean-field approximation of the attractive molecular interaction, leading to expect the invalid physical meanning of Eqs. (1) and (2) for $\Delta \tau^{*} \sim 0.3-1$ [see for example the similar (master) Fig. 2 in Ref. 
[40]). Therefore, it was clearly shown from Fig. 1 that the applicability range of the master crossover functions corresponds to $\xi \gtrsim 2.5 \mathrm{~nm}$ (leading to $\frac{\xi}{\alpha_{c}}>3-5$, i.e., $\frac{\xi}{r_{e}^{\mathrm{LJ}}}>6-10$ and $\Delta \tau^{*} \lesssim 0.02\left(\simeq \mathcal{L}_{\mathrm{EAD}}^{\mathrm{Xe}}\right)$, typically, in the xenon case $)$.

\section{Fluid Turbidity from OZ Theory}

The scattered light intensity is caused by the Rayleigh scattering from large density fluctuations of size $\xi$. From OZ theory, it can be written as follows

$$
I_{\mathrm{OZ}}(q)=\frac{A_{\tau} \kappa_{T} \sin ^{2} \Phi}{1+(q \xi)^{2}}
$$

where $q=\frac{4 \pi n}{\lambda_{0}} \sin \frac{\theta}{2}$ represents the amplitude of the transfer wave vector between incident and scattering wave vectors. $\lambda_{0}$ is the light wavelength in vacuum, $n$ is the refractive index of the fluid, and $\theta$ is the scattering angle. $\Phi$ is the angle between the polarization wave vector of the incident light and the scattering wave vector.

$A_{\tau}=A_{0} k_{B} T \sim\left[\frac{\text { energy }}{\text { (length })^{4}}\right]$, where $k_{B}$ is the Boltzmann constant and where $A_{0} \sim$ [length $]^{-4}$, can be written as

$$
A_{0}=\frac{\pi^{2}}{\lambda_{0}^{4}}\left(\rho \frac{\partial\left(n^{2}\right)}{\partial \rho}\right)_{T}^{2}=\frac{\pi^{2}}{\lambda_{0}^{4}}\left[\frac{\left(n^{2}-1\right)\left(n^{2}+2\right)}{3}\right]^{2}
$$

$A_{0}$ accounts for geometrical factors and light-fluid scattering cross section calculated from the Lorentz-Lorenz approximation of the effective local field, i.e., introducing the related critical value $L L_{c}=\frac{M_{\mathrm{mol}}}{\rho_{c}} \frac{n_{c}^{2}-1}{n_{c}^{2}+2}$ for the density expansion of the Lorentz-Lorenz function.

The turbidity calculated by Puglielli and Ford [24] corresponds to the integral of Eq. (3) over all the scattering angles and writes:

$$
\tau_{\mathrm{PF}}=\pi A_{0} k_{B} T_{c}\left(1+\Delta \tau^{*}\right) \kappa_{T} F(a),
$$

where the universal function $F(a)$ is given by the following equation

$$
F(a)=\left(\frac{2 a^{2}+2 a+1}{a^{3}}\right) \ln (1+2 a)-2\left(\frac{1+a}{a^{2}}\right)
$$

with $a=2\left(k_{0} \xi\right)^{2}$ and $k_{0}=\frac{2 \pi n}{\lambda_{0}}$ ( $k_{0}$ is the amplitude of the incident light wave vector of order of $10^{-7} \mathrm{~m}^{-1}$ for $\left.\lambda_{0} \sim 630 \mathrm{~nm}\right)$. The function $F(a)$ reaches a constant value $F(a) \propto \frac{8}{3}$ for $a \ll 1$ (i.e., for $\xi$ comparable or smaller than the range of the microscopic interaction forces), leading to $\tau_{\mathrm{PF}} \propto T \kappa_{T}$ far away from the critical temperature. When $a \gg 1, F(a) \propto$ $\frac{2[\ln (2 a)-1]}{a}$. Therefore, the asymptotic singular turbidity predicted by Eq. (5) exhibits an incorrect logarithmic singularity approaching $T_{c}$ (see Ref. [32] and the dicussion below).

For the xenon case, all the quantities involved in the above calculation of the fluid turbidity are given in Table 1, where the only differences with the GC's analysis originate from the critical coordinates of xenon. The evaluation of the error-bar attached to the quantities given in Table 1 can be found in I. 


\section{Comparison with Xenon Data}

\subsection{GC Turbidity Measurements}

The GC data points $\tau_{\text {expt }}$ of xenon turbidity are plotted (full blue stars) in Fig. 2a as a function of $\Delta \tau^{*}$ in $\log -\log$ scale. Our theoretical estimation of $\tau_{\mathrm{PF}}$ using Eqs. (5) and (6), with $\xi$ of Eq. (1) and $\kappa_{T}$ of Eq. (2), corresponds to the continuous black curve in Fig. 2a. The GC fitting analysis is also given as the dotted red curve in Fig. 2a. The corresponding residuals (expressed in \%) are given in the log-linear plot of Fig. 2b. Our estimation of $\tau_{\mathrm{PF}}$ without adjustable parameter is in excellent agreement with the experimental measurements and fitting analysis of GC.

When $T \rightarrow T_{c}$, the very small increase of the residuals is mainly due to the small difference in the respective values of the leading critical exponents $v$ and $\gamma$. In addition, the effect of the uncertainty $(\simeq 0.5 \mathrm{mK})$ in the experimental determination of $T_{c, \mathrm{GC}}$ approaching the critical temperature, is illustrated by the full blue squares in Fig. 2b, which correspond to the related behaviors of the residuals due to a $T_{c, \mathrm{GC}}$-change of $+0.5 \mathrm{mK}$. We have also reported the new residuals (full red triangles) due to a change of $-0.00025 \mathrm{~cm}^{-1}$ (i.e., $\simeq-0.6 \%$ ) in the initial calibration value $\left(\tau\left(T_{o r}\right)=0.041 \mathrm{~cm}^{-1}\right.$, see Table 1 and I).

In the temperature range $T-T_{c} \gtrsim 7 \mathrm{~K}$ of Fig. $2 \mathrm{~b}$, increasing of the residuals is due to the significant decreasing of the xenon turbidity when $T-T_{c}$ increases. Indeed, accurate measurements of xenon turbidity in this temperature range require a xenon sample cell of optical path larger than a few centimeters.

Nevertheless, we believe that the OZ approach of xenon turbidity with Eq. (5), incorporating the $\xi$ and $\kappa_{T}$ values obtained from theoretical Eqs. (1) and (2) without adjustable parameter, is adequate in the $T-T_{c} \lesssim 10 \mathrm{~K}$ range covered by the GC measurements. The following section shows that the $\Delta$-contribution to the turbidity behavior is the main contribution in the GC temperature range.

\subsection{Asymptotic Singular Behavior of Turbidity}

The estimation of $\tau$ can now be revisited with the objective to illustrate the role of the Ising-like parameters characterizing the asymptotic singular behavior within the PAD. Indeed, for the Ising-like asymptotic limit $\Delta \tau^{*} \rightarrow 0$, the contributions of the confluent singularities associated to the lowest exponent $\Delta$ can be neglected, leading to $L_{\Delta}\left(\Delta \tau^{*}\right) \approx 1, K_{\Delta}\left(\Delta \tau^{*}\right) \approx$ 1 , and $T=T_{c}\left(1+\Delta \tau^{*}\right) \approx T_{c}$ in the previous equations. Any theoretical asymptotic approach (labelled $\mathrm{X}$ ) estimating the fluid turbidity is then restricted to the knowledge of $\xi=\xi_{0}^{+}\left(\Delta \tau^{*}\right)^{-v}$ and $\kappa_{T}=\Gamma_{0}^{+}\left(\Delta \tau^{*}\right)^{-\gamma}$ and can only add the universal contribution of the pair $\left\{C_{\infty}^{+}, \eta\right\}$ in order to satisfy the two-scale-factor universality (see above). Therefore, Ising-like turbidity must take the general asymptotic form [32,33]

$$
\tau_{a s, \mathrm{X}}=\frac{\tau_{0}\left(\Delta \tau^{*}\right)^{-\gamma}}{y_{v}^{2}} H_{a s, \mathrm{X}}\left(\eta, y_{v}\right)
$$

Equation (7) introduces the universal quantity $y_{v}=k_{0} \xi_{0}^{+}\left(\Delta \tau^{*}\right)^{-v}$ where the subscript $v$ recalls for a Ising universal quantity only characterized by $\left\{\xi_{0}^{+}, v\right\}$. The temperature independent quantity $\tau_{0}=\pi A_{0} k_{B} T_{c} \Gamma_{0}^{+} \sim[\text { length }]^{-1}$ is a fluid dependent quantity, only proportional to $\Gamma_{0}^{+}$. In Eq. (7), the universal scaling function $H_{a s, \mathrm{X}}\left(\eta, y_{\nu}\right)$ accounts for the explicit contribution of $\eta \neq 0$ and depends on the used approximant form for the universal scaling function $G(x) \sim C_{\infty}^{+} x^{\eta-2}$ when $x \gg 1[32,33]$. Now, the explicit derivation of the 


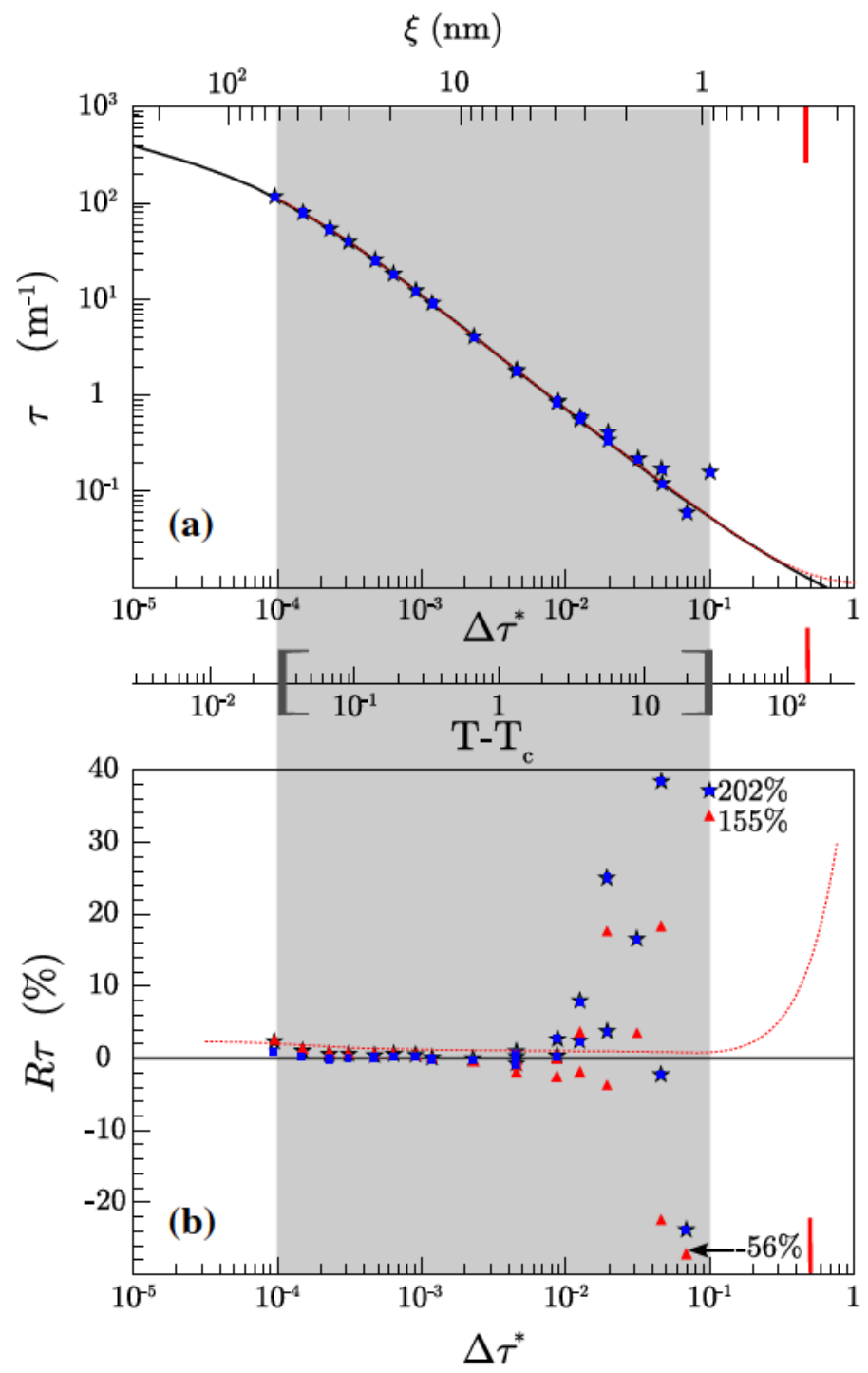

Fig. 2 (a) Log-log plot of $\tau$ (expressed in $\mathrm{m}^{-1}$ ) of xenon along the critical isochore, as a function of $\Delta \tau^{*}$ (lower horizontal axis), $\xi$ (upper horizontal axis) expressed in $\mathrm{nm}$, and $T-T_{c}$ (medium horizontal axis) expressed in $\mathrm{K}$ with $T_{c, \mathrm{GC}}=289.79 \mathrm{~K}\left(16.64^{\circ} \mathrm{C}\right)$ (see I). Full (blue) stars $\mathrm{GC} \tau$-measurements. Full (black) curve from Eq. (5). Dotted (red) curve from I. (b) log-linear plot of $R \tau(\%)=100 \times\left[\frac{\tau}{\tau_{\mathrm{OZ}}}-1\right]$ (expressed in \%) from reference to $\tau_{\mathrm{PF}}$ of Eq. (5); Full (blue) stars with a $T_{c, \mathrm{GC}}$-change of $0.5 \mathrm{mK}$ (see text); Full (red) triangles with a $\tau\left(T_{o r}\right)$-change of $-0.00025 \mathrm{~cm}^{-1}$ (see text) (Color figure online)

asymptotic $\left\{C_{\infty}^{+}, \eta\right\}$-contribution made in conformity with the two-scale universality can be illustrated writting Eq. (7) as follows

$$
\tau_{a s, \mathrm{X}}=\frac{\tau_{0}}{\left(k_{0} \xi_{0}^{+}\right)^{2}}\left(\Delta \tau^{*}\right)^{-\gamma+2 v} H_{a s, \mathrm{X}}\left(\eta, y_{\nu}\right)
$$




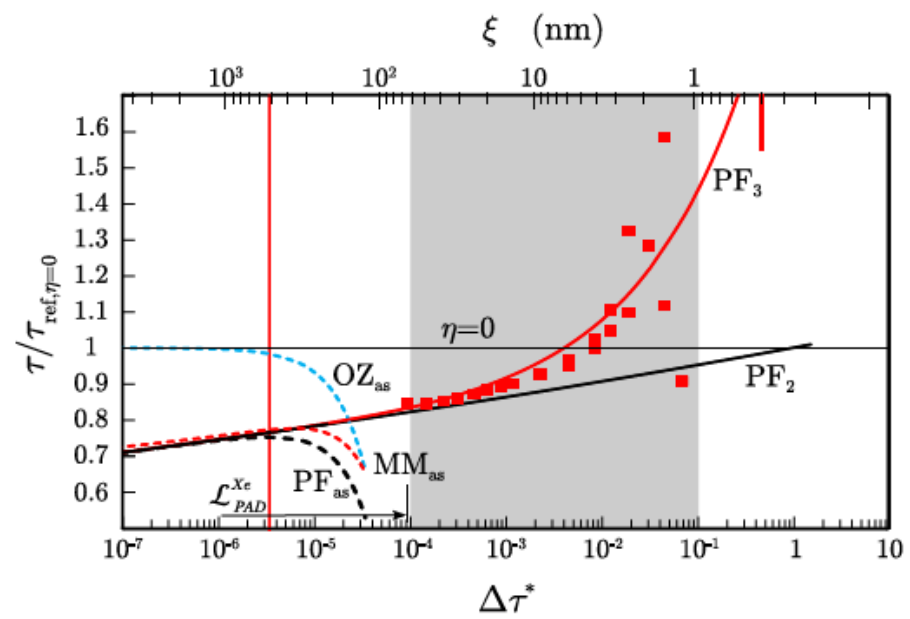

Fig. 3 Log-linear scale: Normalized turbidity $\frac{\tau}{\tau_{\text {ref, }, \eta=0}}$ as a function of $\Delta \tau^{*}$ (see text; horizontal 1-axis corresponds to $\eta=0$ ). Blue tireted curve (label $\left.\mathrm{OZ}_{a s}\right): \frac{\tau_{a s, \mathrm{OZ}}}{\tau_{\mathrm{ref}, \eta=0}}=\frac{L-1}{H_{\mathrm{PF}}\left(y_{v}\right)}$ with $\xi=\xi_{0}^{+}\left(\Delta \tau^{*}\right)^{-v}$ and $\kappa_{T, \mathrm{OZ}}=\Gamma_{0}^{+}\left(\Delta \tau^{*}\right)^{-2 v}$ (exact $\mathrm{OZ}$ theory). Black tireted curve (label $\left.\mathrm{PF}_{a s}\right): \frac{\tau_{a s, \mathrm{PF}}}{\tau_{\mathrm{ref}, \eta=0}}=\left(\Delta \tau^{*}\right)^{-\gamma+2 v}$. Black full curve (label $\mathrm{PF}_{2}$ ): $\frac{\tau_{\mathrm{PF}, v, \gamma}}{\tau_{\mathrm{ref}, \eta=0}}=\left(\Delta \tau^{*}\right)^{-\gamma+2 v}$. Red full curve (label $\mathrm{PF}_{3}$ ): $\frac{\tau_{\mathrm{PF}}}{\tau_{\mathrm{ref}, \eta=0}}=$ $\left(1+\Delta \tau^{*}\right)\left(\Delta \tau^{*}\right)^{-\gamma+2 v} F\left(\Delta \tau^{*}\right) \frac{H_{\mathrm{PF}}(y)}{L-1}$ with $\xi$ and $\kappa_{T}$ of Eqs. (1) and (2), respectively. Red full square: $\frac{\tau_{\exp , \mathrm{GC}}}{\tau_{a s, \eta=0}}$ from GC turbidity measurements in I. (labels are given in other figures) (Color figure online) where $\frac{\tau_{0}}{\left(k_{0} \xi_{0}^{+}\right)^{2}} \propto \frac{\Gamma_{0}^{+}}{\left(\xi_{0}^{+}\right)^{2}}$. The product $\left(\Delta \tau^{*}\right)^{-\gamma+2 v} H_{a s, \mathrm{X}}\left(\eta, y_{v}\right)$ is universal. For $y_{v} \rightarrow \infty$, $H_{a s, \mathrm{X}}\left(\eta, y_{v}\right) \sim\left(y_{v}^{2}\right)^{-\frac{\eta}{2}}$, leading to the expected saturated Ising-like turbidity $(\tau \sim$ constant $)$ approaching $T_{c}$. The saturated turbidity is thus proportional to the amplitude ratio $\frac{\Gamma_{0}^{+}}{\left(\xi_{0}^{+}\right)^{2-\eta}}$, i.e., proportional to the physical leading amplitude $\hat{D}_{\rho, 0}$ of the Ising-like density-density correlation function $\chi_{\rho}(q, \xi=\infty)=\frac{\hat{D}_{\rho, 0}}{q^{2-\eta}}$ in momentum space at the critical point (see above). Such a result satisfies the two-scale factor universality through $Q_{3}=\frac{\hat{D}_{\rho, 0}\left(\xi_{0}^{+}\right)^{2-\eta}}{\rho_{c}^{2} \Gamma_{0}^{+}}$. The validity of this result is however restricted to a near critical temperature range, as discussed below introducing Fig. 3.

Before that, Puglielli and Ford Eq. (5) must be rewritten as follows

$$
\tau_{\mathrm{PF}}=\frac{\tau_{0}\left(1+\Delta \tau^{*}\right)\left(\Delta \tau^{*}\right)^{-\gamma} K_{\Delta}\left(\Delta \tau^{*}\right)}{y^{2}} H_{\mathrm{PF}}(y)
$$

introducing then the universal quantity $y=k_{0} \xi$, such as $a=2 y^{2}$, and the universal function $H_{\mathrm{PF}}(y)=\frac{1}{8 y^{4}}\left(8 y^{4}+4 y^{2}+1\right) \ln \left(1+4 y^{2}\right)-4 y^{2}\left(1+2 y^{2}\right)$, such as $F(a)=\frac{H_{\mathrm{PF}}(y)}{y^{2}}$. In the GC temperature range, we have previously noted that Eq. (8) needs the complete knowledge of the crossover forms for $\xi$ and $\kappa_{T}$, including $L_{\Delta}\left(\Delta \tau^{*}\right), K_{\Delta}\left(\Delta \tau^{*}\right)$ and $T=T_{c}\left(1+\Delta \tau^{*}\right)$, to calculate $y, H_{\mathrm{PF}}(y)$, and finally $\tau_{\mathrm{PF}}$.

However, for $\Delta \tau^{*} \rightarrow 0$, Eq. (9) takes the restricted asymptotic form

$$
\tau_{a s, \mathrm{PF}}=\frac{\tau_{0}}{\left(k_{0} \xi_{0}^{+}\right)^{2}}\left(\Delta \tau^{*}\right)^{-\gamma+2 v} H_{a s, \mathrm{PF}}\left(y_{\nu}\right)
$$


where $H_{a s, \mathrm{PF}}\left(y_{v}\right)=\ln \left[\left(2 y_{v}\right)^{2}\right]-1=L-1$, with $L=\ln \left[\left(2 y_{v}\right)^{2}\right]$. Now, Eq. (9) only needs $\xi$ and $\kappa_{T}$ pure power laws, limiting the validity range of the calculated turbidity that behaves asymptotically with the incorrect logarithmic singularity $H_{a s, \mathrm{PF}}(y) \sim \ln \left(y_{v}^{2}\right)$ approaching $T_{c}$. In spite of this incorrect asymptotic behavior, the practical condition $\gamma \neq 2 v$ corresponds to apply $\eta \neq 0$ in the complete temperature range, leading to the explicit power law term $\left(\Delta \tau^{*}\right)^{-\gamma+2 v}=\left(\Delta \tau^{*}\right)^{\eta v}$ in Eq. (9).

It is then also useful to calculate the turbidity resulting from the exact $\mathrm{OZ}$ condition $\gamma=2 v$, i.e., the turbidity of the OZ-like fluid satisfying $\eta=0$ in the complete temperature range. In such a case, adding $\left(1+\Delta \tau^{*}\right)$ due to $T$, Eq. (10) provides the following reference turbidity

$$
\tau_{\text {ref }, \eta=0}=\frac{\tau_{0}\left(1+\Delta \tau^{*}\right)}{\left(k_{0} \xi_{0}^{+}\right)^{2}} H_{\mathrm{PF}}\left(y_{v}\right)
$$

where $H_{\mathrm{PF}}\left(y_{v}\right)$ is still calculated using $\xi=\xi_{0}^{+}\left(\Delta \tau^{*}\right)^{-v}$, while $\kappa_{T}$ follows the incorrect pure power law $\kappa_{T, \mathrm{OZ}}=\Gamma_{0}^{+}\left(\Delta \tau^{*}\right)^{-2 v}$ resulting from $\kappa_{T} \propto \xi^{2}$. In exact OZ theory, as $\Gamma_{0}^{+}$is a function of $\xi_{0}^{+}$, the fluid turbidity is only characterized by the single amplitude-exponent pair $\left\{\xi_{0}^{+}, v\right\} \cdot \tau_{\text {ref }, \eta=0}$ always presents the incorrect logarithmic singularity $\tau_{a s, \eta=0}=\frac{\tau_{0}}{\left(k_{0} \xi_{0}^{+}\right)^{2}}(L-1) \sim \ln \left(y_{v}^{2}\right)$, similar to the one of $\tau_{a s, \mathrm{PF}}$. The ratio $\frac{\tau_{a s, \eta=0}}{\tau_{\text {ref }, \eta=0}}=\frac{L-1}{H_{\mathrm{PF}}\left(y_{\nu}\right)} \rightarrow 1$ eliminates the logarithmic singularity when $\Delta \tau^{*} \rightarrow 0$. This asymptotic result corresponds to the blue tireted curve labeled $\mathrm{OZ}_{a s}$ in the diagram $\frac{\tau}{\tau_{\mathrm{ref}, \eta=0}} ; \Delta \tau^{*}$ of Fig. 3. The horizontal axis $\frac{\tau}{\tau_{\text {ref }, \eta=0}}=1$ corresponds to $\eta=0$. For a true Ising-like fluid, the respective contributions due to $\eta \neq 0$ and $\Delta$ are then easy to illustrate in Fig. 3, at each temperature distance to $T_{c}$.

For the Ising-like limiting range $\Delta \tau^{*} \leq 10^{-6} \ll \mathcal{L}_{\mathrm{PAD}}^{\mathrm{Xe}}$ where the scaling law $\eta v=$ $-\gamma+2 v$ can be used to estimate $\eta, \frac{\tau_{a s, \mathrm{PF}}}{\tau_{\mathrm{ref}, \eta=0}}=\left(\Delta \tau^{*}\right)^{-\gamma+2 v} \frac{L-1}{H_{\mathrm{PF}}\left(y_{v}\right)} \rightarrow\left(\Delta \tau^{*}\right)^{-\gamma+2 v}$ (see the black tireted curve $\mathrm{PF}_{a s}$ in Fig. 3). In this asymptotic range, the relative difference between the $\mathrm{OZ}_{a s}$ and $\mathrm{PF}_{a s}$ curves reveals that the amplitude of the contribution due to $\eta \neq 0$ is larger than $25 \%$ and increases when $\Delta \tau^{*} \rightarrow 0$. Indeed, if only the pure power laws of $\xi$ and $\kappa_{T}$ are used for the complete $\Delta \tau^{*}$ range, Eq. (8) takes the simplified form $\tau_{\mathrm{PF}, v, \gamma}=\frac{\tau_{0}\left(1+\Delta \tau^{*}\right)}{\left(k_{0} \xi_{0}^{+}\right)^{2}}\left(\Delta \tau^{*}\right)^{-\gamma+2 v} H_{\mathrm{PF}}\left(y_{\nu}\right)$ (labeled by the subscript PF, $\left.v, \gamma\right)$. Accordingly, $\frac{\tau_{\mathrm{PF}, v, \gamma}}{\tau_{\mathrm{ref}, \eta=0}}=\left(\Delta \tau^{*}\right)^{\eta v}$ whatever $\Delta \tau^{*}$, as illustrated by the black curve labeled $\mathrm{PF}_{2}$ in Fig. 3 . A pure fluid turbidity without contribution of the confluent singularities associated to $\Delta$ is only characterized by both pairs $\left\{\xi_{0}^{+}, \nu\right\}$ and $\left\{\Gamma_{0}^{+}, \gamma\right\}$. The subscript 2 recalls for a fluid characterized by only two leading amplitudes $\left(\xi_{0}^{+}\right.$and $\left.\Gamma_{0}^{+}\right)$over the complete temperature range. Consequently, as $\Delta \tau^{*}$ decreases, the increasing difference between 1-axis and $\mathrm{PF}_{2}$ curve is a measure of $\eta \neq 0$.

However, as mentioned previously, the above asymptotic singular behavior where the turbidity is calculated from the PF functional form, involves a fluid characterization without conformity with the two-scale factor universality. To illustrate the amplitude effect of the asymptotic singular behavior of the true Ising-like turbidity, we use as a typical example the Martin-Mayor et al's turbidity results of Ref. [33] obtained by Monte Carlo simulation of a simple cubic Ising lattice. Such results have validated the analytic derivation of the saturated turbidity at $T_{c}, \rho_{c}$ initially proposed by Ferrell [32]. Here using the functional form $H_{a s, \mathrm{MM}}\left(\eta, y_{v}\right)=2 C_{\infty}^{+}\left\{\left(4 y_{v}^{2}\right)^{\frac{\eta}{2}}\left[\frac{\eta^{2}+2 \eta+8}{\eta(\eta+2)(\eta+4)}\right]-\frac{1}{\eta}+\frac{K}{C_{\infty}^{+}}\right\}$given by Eq. (34) in Ref. [33], the asymptotic behavior of the corresponding ratio $\frac{\tau_{a s, \mathrm{MM}}}{\tau_{\mathrm{ref}, \eta=0}}=\left(\Delta \tau^{*}\right)^{-\gamma+2 v} \frac{H_{a s, \mathrm{MM}}\left(\eta, y_{v}\right)}{H_{\mathrm{PF}}\left(y_{v}\right)}$ 
is represented by the red tireted curve labelled $\mathrm{MM}_{a s}$ in Fig. 3 (using $K=0.16$ ). When $\Delta \tau^{*} \rightarrow 0$, the increasing difference from $\mathrm{MM}_{a s}$ and $\mathrm{PF}_{a s}$ curves reveals the increasing $\left\{C_{\infty}^{+}, \eta\right\}$-contribution to the turbidity of the Ising-like fluid which complies with the twoscale factor universality.

Finally, it remains to illustrate the $\Delta$-contribution to the fluid turbidity when the temperature distance to the critical temperature increases well beyond the PAD, i.e., $\Delta \tau^{*}>$ $10^{-5}$. Using Eqs. (1) and (2), $\frac{\tau_{\mathrm{PF}}}{\tau_{\mathrm{ref}, \eta=0}}=\left(\Delta \tau^{*}\right)^{\eta \nu} \frac{K\left(\Delta \tau^{*}\right)}{\left[L\left(\Delta \tau^{*}\right)\right]^{2}} \frac{H_{\mathrm{PF}}(y)}{H_{\mathrm{PF}}\left(y_{\nu}\right)}$, leading to the red full curve labelled $\mathrm{PF}_{3}$ in Fig. 3. Now, the subscript 3 recalls for a fluid characterized by two leading amplitudes $\left(\xi_{0}^{+}\right.$and $\left.\Gamma_{0}^{+}\right)$and one first-order amplitude $\left(a_{\chi}^{1,+}\right)$ acting as a single crossover parameter over the complete temperature range. As $\Delta \tau^{*}$ increases, the increase of the $\Delta$-contribution is thus measured by the increasing difference from $\mathrm{PF}_{3}$ and $\mathrm{PF}_{2}$ curves in Fig. 3. The agreement with $\frac{\tau_{\exp }}{\tau_{\mathrm{ref}, \eta=0}}$ obtained from GC turbidity data (red squares in Fig. 3) is noticeable, demonstrating the interest of the present work made without any adjustable parameter as a correct answer to the GC conclusive sentence in I.

An additional remark can be formulated from a hypothetical turbidity measurement, $0.3 \%$ precision, performed at $1 \mathrm{mK}$ temperature distance above $T_{c}$, i.e., $\Delta \tau^{*} \sim 3.45 \times 10^{-6}$ in xenon case (see red vertical line in Fig. 3). At such a finite temperature distance well-inside the PAD extension, the $\Delta$-contribution due to the confluent corrections to scaling is lower than the experimental precision and can be certainly neglected. From Puglielli and Ford result, $\frac{\tau_{a s, \mathrm{PF}}}{\tau_{\text {ref }, \eta=0}}=0.766 \pm 0.019$, i.e., $\sim \pm 2.5 \%$ error-bar (with central value $v=0.63$ and accounting for the $\eta$-theoretical error-bar, $\left.\eta=0.0336_{-0.00250}^{+0.00294}\right)$. From Martin-Mayor result, $\frac{\tau_{a s, \mathrm{MM}}}{\tau_{\mathrm{ref}, \eta=0}}=0.774 \pm 0.025$ (increasing to $\pm 3.5 \%$ error-bar after adding $\pm 1 \%$ error-bar from the theoretical estimation of $\left.C_{\infty}^{+}\right)$. Therefore, when the two asymptotic power laws $\xi=$ $\xi_{0}^{+}\left(\Delta \tau^{*}\right)^{-v}$ and $\kappa_{T}=\Gamma_{0}^{+}\left(\Delta \tau^{*}\right)^{-\gamma}$ are known, the agreement with the experimental value of the turbidity at $T-T_{c}=1 \mathrm{mK}$ is a significant probe of the related value $\eta=2-\frac{\gamma}{v}$, whatever the theoretical functional form of the turbidity. However, as the residual $100 \times\left(\frac{\tau_{a s, \mathrm{MM}}}{\tau_{a s, \mathrm{PF}}}-1\right)$ is only $+1 \%$, the distinction between the contributions due to the respective ratios $\frac{\Gamma_{0}^{+}}{\left(k_{0} \xi_{0}^{+}\right)^{2}}$ (involved through the Puglielli and Ford Eq. (9)) and $\frac{\Gamma_{0}^{+}}{\left(k_{0} \xi_{0}^{+}\right)^{2-\eta}}$ (involved through the MartinMayor et al. Eq. (7)) remains not significant at such a small temperature distance. We note that the difficulty can be partly solved when an additional singular property is measured, especially the top-shape $\Delta \widetilde{\rho}_{L V}=B\left(\Delta \tau^{*}\right)^{\beta}$ of the coexistence curve, which gives access to the ratio $\frac{\Gamma_{0}^{+}}{\left(\xi_{0}^{+}\right)^{3}}$ through the non-dimensional amplitude combination $\left(\xi^{+}\right)^{-d} \frac{\Gamma^{+}}{B^{2}}$. In Sect. 6, we show that the two leading amplitudes $\Gamma^{+}$and $B$ are thus well-adapted for the thermodynamic characterization of the fluid, while the role of turbidity for the correlation characterization of the fluid is discussed in Ref. [41], analyzing recent measurements of $\mathrm{SF}_{6}$ turbidity in the close vicinity of its liquid-gas critical point.

\subsection{Correlation Length}

We have reported in the lines $\sharp 1$ to $\sharp 8$ of Table 3 the published amplitude/exponent values of the leading power law $\xi=\xi_{0}^{+}\left(\Delta \tau^{*}\right)^{-v}$, which have been used in the indirect fitting of the results provided by (static and dynamic) Rayleigh-Brillouin methods [42-47]. Line $\sharp 9$ 
results from the GC fitting analysis of their turbidity data with fixed values of the exponents in the following two-term Wegner expansion

$$
\xi=\xi_{0}^{+}\left(\Delta \tau^{*}\right)^{-v}\left[1+a_{\xi}^{1,+}\left(\Delta \tau^{*}\right)^{\Delta}\right]
$$

In addition, we have also given in lines $\sharp 10$ and $\sharp 11$ the $\xi_{0}^{+}, v$ values for two fixed pure power laws. Indeed, such pure power laws are fixed in the fitting analyses of the shear viscosity [48], the thermoacoustic boundary layers [36], and the bulk viscosity [49] of critical xenon. i.e., experiments which cover the extended temperature range $5 \times 10^{-7} \leq \Delta \tau^{*} \leq$ $10^{-1}$. These applications then extend significantly the GC temperature range, especially by two supplementary decades approaching the critical temperature. Finally, line $\sharp 12$ gives the related values for the two-term form of Eq. (1), whose validity is restricted to the PAD extension.

The log-lin plots of Fig. 4a, b show the corresponding residuals $R \xi(\%)=100 \times\left(\frac{\xi}{\xi_{\text {cal }}}-1\right)$ (expressed in \%) from reference to $\xi_{\text {cal }}$ calculated using Eq. (1). The curves labeled 1 to 8 of part (a) illustrate the significant dispersion (larger than $10 \%$ ) of the results (lines $\sharp 1$ to $\sharp 8$ of Table 3) provided by using (static and dynamic) Rayleigh-Brillouin methods (including the first fitting analysis [47] of the correlation length measurements made by using the precise differential technique and apparatus). The residuals with the data measurements performed by Smith et al. [42] are represented by the full black circles. This Fig. 4a gives clear evidence that the highly correlated values of $v$ and $\xi_{0}^{+}$are not obtained with the required precision from all these optical measurements performed at finite distance to $T_{c}$. In (magnified) part (b) of Fig. 4, the lines (labeled 9 to 11) correspond to the residuals (then lowered at the $1-2 \%$-level) using Eq. (11) with the Ising-like parameters of lines $\sharp 9$ to $\sharp 11$ of Table 3). In spite of the questionable justification of the universal features related to the lowest order of the Ising-like Wegner expansions at large distance from $T_{c}$, we note a satisfactory agreement (within the experimental uncertainty of $8 \%$ ) in the reduced temperature range $7 \times 10^{-5} \leq \Delta \tau^{*} \leq 2 \times 10^{-2} \simeq \mathcal{L}_{\mathrm{EAD}}^{\mathrm{Xe}}($ Fig. 5).

Table $3 \sharp 1$ to $\sharp 11$ : Literature exponent-amplitude values for fitting Eq. (11) of xenon correlation length when $\tau \rightarrow 0$ along the critical isochore (n.u.: not used)

\begin{tabular}{lllll}
\hline$\sharp$ & $v$ & $\xi_{0}^{+}(\mathrm{nm})$ & $a_{\xi}^{1,+}\left(\Delta \tau^{*}\right)^{\Delta}$ & Ref. \\
\hline 1 & 0.60 & 0.302 & n.u. & {$[43]$} \\
2 & $0.58 \pm 0.05$ & $0.3 \pm 0.01$ & n.u. & {$[42]$} \\
3 & $0.57 \pm 0.03$ & 0.307 & n.u. & {$[44]$} \\
4 & $0.64 \pm 0.02$ & $0.136 \pm 0.006$ & n.u. & {$[45]$} \\
5 & 0.63 & 0.2 & n.u. & {$[42,46]$} \\
6 & 0.63 & 0.167 & n.u. & {$[46]$} \\
7 & 0.58 & 0.232 & n.u. & {$[46]$} \\
8 & 0.62 & 0.193 & n.u. & {$[47]$} \\
9 & 0.63 & $0.184 \pm 0.009$ & $0.55\left(\Delta \tau^{*}\right)^{0.5}$ & {$[1]$} \\
10 & 0.63 & $0.184 \pm 0.009$ & n.u. & {$[1,48]$} \\
11 & 0.63 & $0.1866 \pm 0.0010$ & n.u. & {$[1,36,49]$} \\
12 & 0.6303875 & 0.184531 & $0.55\left(\Delta \tau^{*}\right)^{0.50189}$ & This work \\
\hline
\end{tabular}

$\sharp 12$ : Corresponding asymptotic result from Eq. (1) restricted to the two-term contribution of Eq. (11) 


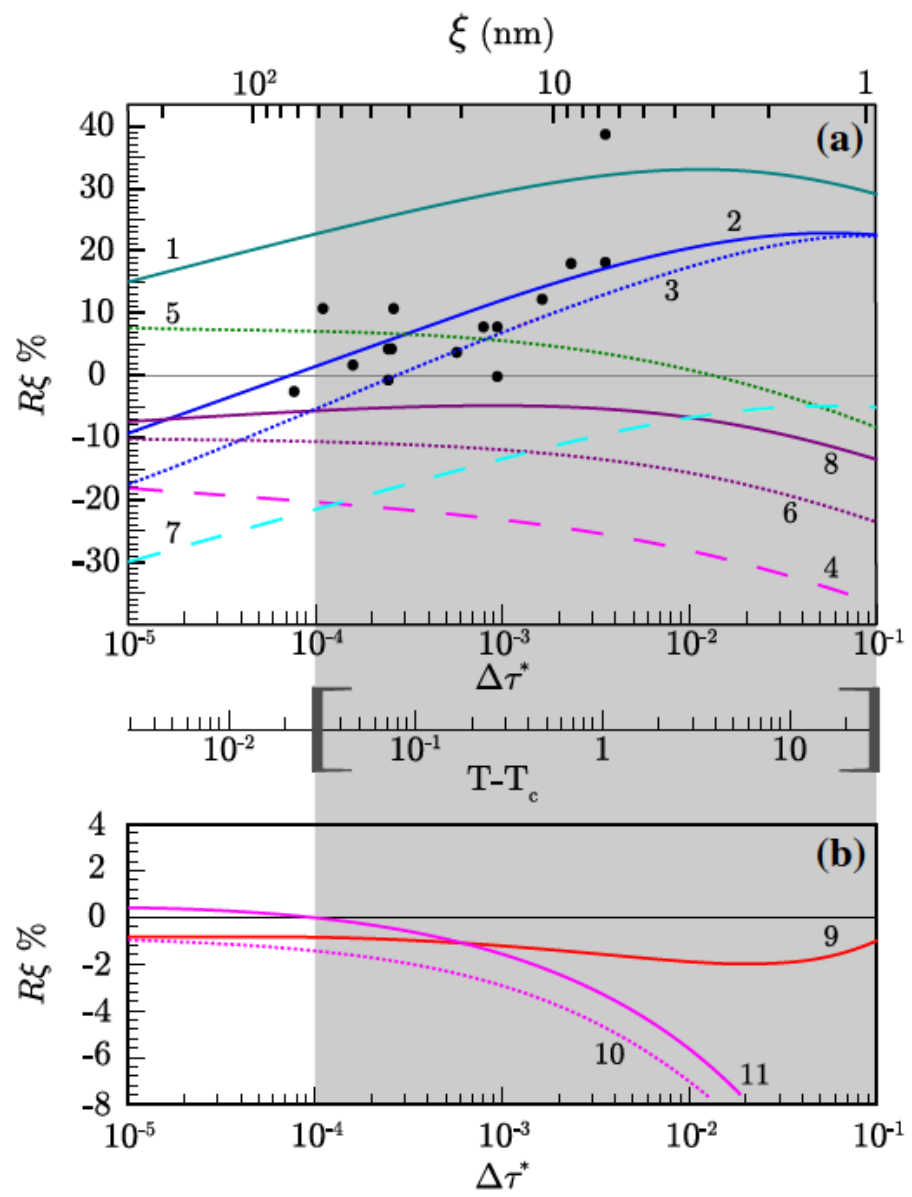

Fig. 4 Log-linear scale: residuals $R \xi(\%)=100 \times\left(\frac{\xi}{\xi_{\text {cal }}}-1\right)$ (expressed in $\%$ ) for the values of the xenon correlation length $\xi$ as a function of $\Delta \tau^{*}$, from reference to the calculated value $\xi_{\text {cal }}$ using Eq. (1). a Results published earlier than the GC measurements: Full black circles experimental data of Ref [42]. Curves labeled 1 to 8: pure power law $\xi=\xi_{0}^{+}\left(\Delta \tau^{*}\right)^{-v}$ with adjustable exponent and amplitude values given in $\sharp 1$ to $\sharp 8$ of Table 3, respectively. b: Results obtained by GC; Curves labeled 9 to 11: Eq. (11) (red line) with Ising-like exponent values fixed in $\sharp 9$ to $\sharp 11$ of Table 3 , respectively (Color figure online)

\subsection{Isothermal Compressibility}

The detailed analysis of the $\kappa_{T}\left(\Delta \tau^{*}\right)$ measurements can be found in Ref. [15]. Here, through the $\gamma ; \Gamma^{+}$values of Refs. $[1,12,13,15,50-56]$ reported in lines labeled $\sharp 1$ and $\sharp 10$ of Table 4, we only underline the excellent agreement between our PAD-restricted, two-term Wegner expansion

$$
p_{c \kappa_{T}}=\Gamma^{+}\left(\Delta \tau^{*}\right)^{-\gamma}\left[1+a_{\chi}^{1,+}\left(\Delta \tau^{*}\right)^{\Delta}\right]
$$

(line labeled $\sharp 10$ ) of the isothermal compressibility calculated from Eq. (2) and the related leading power laws published in the literature, especially when the Ising-like value of $\gamma$ covers the small range $1.230 \leq \gamma \leq 1.242$ (lines $\sharp 5$ and $\sharp 10$ ). It is then interesting to note that the scaled forms of the equation of state $[52,53]$ can be controled by calculating its free 
Table 4 Exponent-amplitude values for fitting Eq. (12) of the isothermal compressibility data when $\Delta \tau^{*} \rightarrow 0$ along the critical isochore of xenon. $\sharp 1$ : from GC turbidity measurements

\begin{tabular}{llllll}
\hline$\sharp$ & $\gamma$ & $\Gamma^{+}$ & $\Delta$ & $a_{\chi}^{1,+}$ & Ref. \\
\hline 1 & 1.241 & $0.0577 \pm 0.001$ & 0.496 & $1.29 \pm 0.03$ & {$[1]$} \\
2 & 1.23 & $0.062 \pm 0.006$ & $0.062 \pm 0.006$ & $0.062 \pm 0.006$ & {$[50]$} \\
3 & 1.24 & $0.058 \pm 0.002$ & n.u. & & {$[50,51]$} \\
4 & 1.24 & 0.5779 & n.u. & & {$[52,53]$} \\
5 & 1.24194 & $0.057 \pm 0.001$ & 0.491 & 1.1844 & {$[1,13]$} \\
6 & 1.239 & n.a. & 0.51 & 1.08 & {$[1,54]$} \\
7 & 1.237 & $0.0594 \pm 0.0002$ & $0.508(25)$ & n.a. & {$[1,55]$} \\
8 & 1.2396 & $0.0587 \pm 0.0040$ & 0.504 & $1.11 \pm 0.13$ & {$[1,56]$} \\
9 & 1.239 & $0.059 \pm 0.003$ & 0.52 & $0.96 \pm 0.47$ & CPM (this work) \\
$9-$ bis & 1.239 & 0.058 & 0.52 & 1.16 & CMM (this work) \\
10 & 1.2395935 & 0.0578238 & 0.50189 & 1.22646 & MR (this work) \\
\hline
\end{tabular}

$\sharp 2$ and $\sharp 3$ : from Fraunhofer optical measurements of density profiles of [50,51]; na: non available; $\sharp 4$ : Leading values calculated using the restricted cubic model of the scaled equation of state $[52,53] ; \sharp 5$ and $\sharp 8$ : Fitting results of the GC data using different crossover functions; $\sharp 9$ and $\sharp 9$-bis: Results of the CPM and CMM, respectively (see Sect. 6.2 of the text); $\sharp 10$ : Corresponding asymptotic result from Eq. (2)

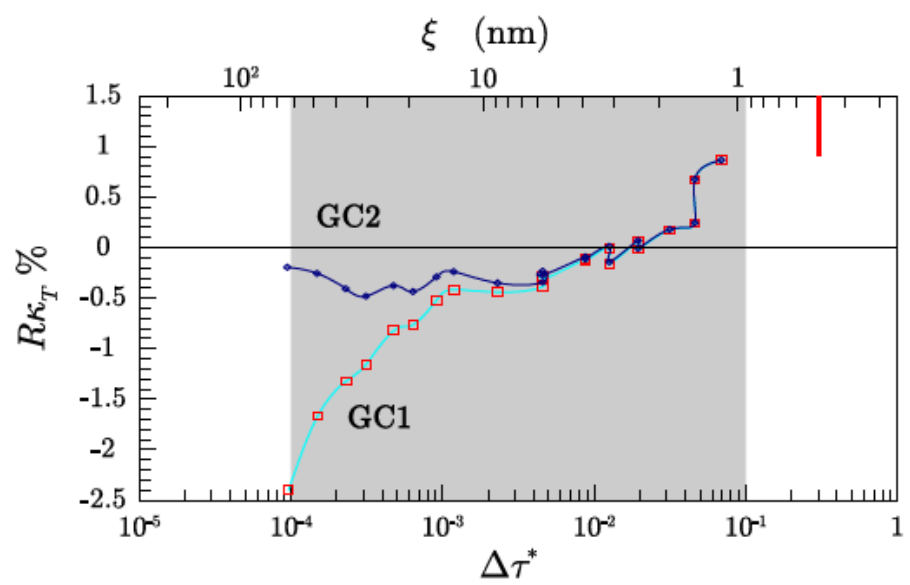

Fig. 5 Log-linear scale: residuals $R_{\kappa_{T}}(\%)=100 \times\left(\frac{\kappa_{T}}{\kappa_{T} \text {,cal }}-1\right)$ (expressed in $\%$ ) for the values of the xenon compressibility $\kappa_{T}$ as a function of $\Delta \tau^{*}$, from reference to the calculated value $\kappa_{T \text {,cal }}$ using Eq. (2). Curves labeled GC1 and GC2: GC experimental data without (GC1) and with (GC2) a $T_{c}$-shift of $0.5 \mathrm{mK}$ (Color figure online)

parameters from the same set of the four critical coordinates defined in Sect. 3. In such a situation, the asymptotic singular behaviors of the xenon properties follow as close as possible the Ising-like singular behaviors fitted from the adjustable theoretical crossover functions (see line $\sharp 5$ and Ref. [13]) or directly calculated using the master crossover functions of the fluid subclass (see line $\sharp 10$ and Ref. [14]).

It is worth noticing that the comparison between the results reported on lines $\sharp 9$ and $\sharp 10$ of Table 4 indicates satisfactory agreement for the asymptotic singular behaviors calculated by the crossover parametric model (CPM) [16] of the equation of state (EOS) and by the 
above crossover function of Eq. (2) issued from the MR scheme. More generally, it was already noted the inherent Ising-like similarity between the MR scheme and the approach based on a phenomenological crossover transformation for a classical Landau expansion of the singular contribution to a free energy density $[17,18]$. Both approaches account for similar asymptotic universal features at the first-order of the confluent singularities, only using three adjustable physical parameters. On such a theoretical basis, we are in position to write the equations that close the unambiguous determination of the CPM free parameters, using the scale factors of the master crossover functions as entry data. It is then expected that the resulting crossover master model (CMM) works without any Ising-like adjustable parameter. Therefore, our following section is focused on the phenomenological key points of the crossover transformation of the equation of state in order to propose a new tool able to estimate the fluid properties in the complete phase surface surrounding the vicinity of its liquid-gas critical point, only knowing the four critical parameters previously introduced in Sect. 3.

\section{Master form of the Crossover Parametric Model}

\subsection{Main Characteristics of CPM}

The CPM developped by Agayan et al [16] results from a generic approach based on a phenomenological crossover transformation for a classical Landau expansion of the singular contribution to a free energy density. This approach was at the origin of the so-called crossover Landau model (CLM) [17,18], where the van der Waals equation was used to develop the parametric form of the equation of state. Due to the initial implementing by Chen et al. $[17,18]$ of a match-point method proposed by Nicoll et al. [19-21], the CPM appears to be conform with the so-called renormalization-group matching technique, while reproducing now the known theoretical values for the universal amplitudes combinations of leading terms of the scaling behaviors and first-order terms of the confluent singularities in Wegner-like expansions.

In a parametric equation-of-state, any point of the phase surface close to the critical point is characterized by the radial variable $r$, which measures the distance to the critical point and the angular variable $\theta$, which represents the density distance to the critical density on a contour of constant $r$. The temperature distance and the density distance of the critical point are thus related to $r$ and $\theta$ by parametrics forms. In addition, these parametric forms contain a crossover function able to represent the phenomenological crossover transformation for a classical Landau expansion of the singular contribution of a free energy. Starting then far from the critical point where it is observed the classical—so called mean field-behavior of the singular energy, crossover occurs when approching the critical point, to finally observe the critical-so called Ising-like-behavior of the singular energy very close to the critical point. In the CPM model, this phenomenological crossover behavior is defined by a function $Y$, formulated to satisfy the following equation

$$
1-(1-\bar{u}) Y=\bar{u}\left(1+\frac{\Lambda^{2}}{\kappa^{2}}\right)^{\frac{1}{2}} Y^{\frac{v}{\Delta_{s}}}
$$

where $\kappa$ is the inverse of the dimensionless correlation length $\xi^{*}$ and $v$ is the corresponding universal critical exponent. $\Delta_{S}$ (in Agayan et al's notations) is identical to the lowest value of the critical exponent $\Delta$ for the confluent singularities of the Wegner expansion (see for example Eqs. (1) and (2)). 
With a parametrization of $\kappa$ as a function of $r$ as

$$
\kappa^{2}(r)=c_{t} r Y^{\frac{2 v-1}{\Delta_{s}}}
$$

the crossover function $Y$, like $\kappa$, are only linear functions of $r$ and independent of $\theta . c_{t}$ is a non-dimensional (fluid-dependent) scale-factor for the reduced temperature distance to $T_{c}$ along the critical isochore. We note also that $Y$ only depends on two crossover variables, $\bar{u}$, and $\frac{\Lambda}{\left(c_{t}\right)^{\frac{1}{2}}}$, which determine the crossover shape and the crossover temperature scale, respectively (see also below).

The non dimensional ordering field $h_{1}$, the non dimensional non-ordering field $h_{2}$, and the critical part $\Delta \Phi$ of the non dimensional thermodynamic potential can thus be described by parametric representations in terms of the variables $r$ and $\theta$ and by appropriate scaling forms of $Y$, as follows

$$
\begin{aligned}
h_{1} & =r^{\frac{3}{2}} Y^{\frac{2 \beta \delta-3}{2 \Delta_{s}} \tilde{l}(\theta)} \\
h_{2} & =r k(\theta) \\
\Delta \Phi & =r^{2} Y^{-\frac{\alpha}{\Delta_{s}}} \tilde{w}(\theta)+\frac{1}{2} B_{c r} r^{2}\left(1-b^{2} \theta^{2}\right)^{2}
\end{aligned}
$$

where $\beta, \delta$, and $\alpha$ are the critical exponents for the top shape of the coexistence curve, the critical isotherm, and the heat capacity at constant volume, respectively. Among the $v, \eta$, $\gamma, \beta, \delta$, and $\alpha$ exponents involved in this phenomenological description, only two remain independent (using for example the additional hyperscaling laws $d v=2-\alpha, \frac{2-\eta}{d}=$ $\frac{\delta-1}{\delta+1}$, and the scaling laws $\gamma=v(2-\eta), \gamma=\beta(\delta-1)$, when $v$ and $\gamma$ are selected as independent). The above functional forms of Eqs. (14) to (16), also introduce the following analytic functions of $\theta$

$$
\begin{aligned}
\tilde{l}(\theta) & =\tilde{l}_{0} \theta\left(1-\theta^{2}\right) \\
k(\theta) & =1-b^{2} \theta^{2} \\
\tilde{w}(\theta) & =\tilde{m}_{0} \tilde{l}_{0}\left(w_{0}+w_{1} \theta^{2}+w_{2} \theta^{4}+w_{3} \theta^{6}+w_{4} \theta^{8}\right)
\end{aligned}
$$

and the fluctuation induced constant

$$
B_{c r}=-2 \tilde{m}_{0} \tilde{l}_{0} w_{0}<0
$$

where appear six universal parameters $\left(b^{2}\right.$ and $w_{i}$, with $\left.i=\{0,4\}\right)$ and two rescaled parameters $\left(\tilde{m}_{0}=m_{0} g^{\beta-\frac{1}{2}}\right.$ and $\left.\tilde{l}_{0}=l_{0} g^{\beta \delta-\frac{3}{2}}\right)$ accounting for the characterization of the physical system. $m_{0}$ and $l_{0}$ are the two system-dependent parameters that determine the asymptotic critical amplitudes to be conform with the two-scale-factor universality (see below). $g=\frac{(\bar{u} \Lambda)^{2}}{c_{t}}$ is the crossover parameter that combine the two previous crossover parameters $\bar{u}$ and $\frac{\Lambda}{\left(c_{t}\right)^{\frac{1}{2}}}$ characterizing the crossover function $Y$. Here it is essential to note that the parameter $b^{2}$ and the five coefficients $w_{i}$ of the parametrized CPM, were chosen such that the asymptotic amplitude ratios between the asymptotic thermodynamic amplitudes $A_{0}^{+}, A_{0}^{-}$, $\Gamma_{0}^{+}, \Gamma_{0}^{-}, B_{0}$, and $D_{0}$ (in Agayan et al's dimensionless notations), agree with the theoretical predictions of the universal features of a Ising-like system [57,58]. Finally, among the six universal asymptotic parameters $b^{2}$ and $w_{i}$, only two are independent.

To describe the one-component fluid crossover using the intensive variables $p$ and $T$ associated to the molar density $n_{\mathrm{mol}}=\frac{N}{N_{A}} \frac{1}{V}$, the classical thermodynamic form of the CPM 
assumes that the scaling fields $h_{1}$ and $h_{2}$ can be expressed in the linear combinations of the dimensionless Gibbs energy density and temperature distances to their respective critical value, i.e., $h_{1}=\Delta g^{*}$ and $h_{2}=\Delta T^{*}+b_{2} \Delta g^{*}$, where $b_{2}$ is the mixing parameter that measures the asymmetry in the slope of the coexistence curve. Here $\Delta T^{*}=1-\frac{T_{c}}{T}=\frac{\Delta \tau^{*}}{1+\Delta \tau^{*}}$ and $\Delta g^{*}=g^{*}-g_{c}^{*}$. The definition of $\Delta g^{*}$, which acts as a conjugated ordering field, needs to recall that the density $g(T, p)=\frac{G(T, p, N)}{V}$ of the Gibbs free energy (distinct from the crossover parameter $g$ ) can be expressed as $g(T, p)=n_{\bar{p}} \mu_{\bar{p}}=n_{\text {mol }} \mu_{\text {mol }}(T, p)$, introducing then the molar chemical potential $\mu_{\mathrm{mol}}(T, p)=N_{A} \mu_{\bar{p}}(T, p)$. Obviously, $\Delta \mu_{\mathrm{mol}}=\mu_{\mathrm{mol}}-$ $\mu_{\mathrm{mol}, c}=N_{A} \Delta \mu_{\bar{p}}$, where $\Delta \mu_{\bar{p}}=\mu_{\bar{p}}-\mu_{\bar{p}, c}$. Moreover, in the related dimensionless form of the CPM, the density of any thermodynamic potential (i.e., an energy per unit of volume) is expressed in units of $p_{c} \equiv \frac{\left(\beta_{c}\right)^{-1}}{\left(\alpha_{c}\right)^{d}}$, while the density of the entropy is expressed in units of $\frac{p_{c}}{T_{c}} \equiv \frac{k_{B}}{\left(\alpha_{c}\right)^{d}}$. Therefore, $g^{*}=\frac{n_{\mathrm{mol}, c} \mu_{\mathrm{mol}}}{p_{c}}$ and $\Delta g^{*}=\frac{n_{\mathrm{mol}, c}}{p_{c}} \Delta \mu_{\mathrm{mol}}$ (with $n_{\mathrm{mol}, c}=\frac{n_{\bar{p}, c}}{N_{A}}$ ). The scaling field $\Delta g^{*}$ can then be related to the previously introduced dimensionless ordering field $\Delta \mu_{\bar{p}}^{*}$ (or $\left.\Delta \tilde{\mu}\right)$ by the equation $\Delta g^{*}=\frac{1}{Z_{c}} \Delta \mu_{\bar{p}}^{*}$ (or $\Delta g^{*}=\Delta \tilde{\mu}$ ). In such a thermodynamic representation, the thermodynamic potential $\Phi$ corresponds to the density $\frac{J}{V}$ of the Grand potential $J=-p V$ and can be written as $\Phi=\frac{p V}{V} \frac{1}{p_{c}}=\frac{j}{p_{c}}=p^{*}$ in dimensionless units. The singular thermodynamic potential is $\Delta \Phi=\Phi-\Phi_{c}=p^{*}-1$. The corresponding conjugated parameters to $h_{1}$ and $h_{2}$ are $\varphi_{1}=-\left(\frac{\partial \Delta \Phi}{\partial h_{1}}\right)_{h_{2}}=\Delta \widetilde{n}_{\mathrm{mol}}-b_{2} \Delta \widetilde{s}$ and $\varphi_{2}=$ $-\left(\frac{\partial \Delta \Phi}{\partial h_{2}}\right)_{h_{1}}=\Delta \widetilde{\boldsymbol{S}}$, respectively. We recall here that the order parameter $\Delta \widetilde{n}_{\mathrm{mol}}=\frac{n_{\mathrm{mol}}}{n_{\mathrm{mol}, c}}-1$ is related to the previous order parameters by $\Delta \widetilde{n}_{\text {mol }}=\Delta \widetilde{\rho}=\frac{\rho}{\rho_{c}}-1$ and $\Delta \widetilde{n}_{\text {mol }}=\frac{1}{Z_{c}} \Delta n_{\bar{p}}^{*}$ (with $\rho=n_{\text {mol }} M_{\text {mol }}=n \bar{p} m \bar{p}$ ). In addition, $\Delta \widetilde{s}=\widetilde{s}-\widetilde{s}_{c}$ is the difference between the dimensionless entropy density $\widetilde{s}=\frac{S}{V} \frac{T_{c}}{p_{c}}$ and its (arbitrary) value $\widetilde{s}_{c}=\frac{S_{c}}{V} \frac{T_{c}}{p_{c}}$ at the critical point (with $\widetilde{s} \equiv s^{*}$ and $\Delta \widetilde{s} \equiv \Delta s^{*}$, where the dimensionless variables with asterisk use $\left(\beta_{c}\right)^{-1}$ and $\alpha_{c}$ as energy and length units). Once $h_{1}, h_{2}$ and $\Delta \Phi=p^{*}-1$ are defined in terms of the parametric variables $r$ and $\theta$, thermodynamic quantities can then be derived using well known thermodynamic relations.

However, in order to maintain Ising-like similarity with the symmetrical $\left(\phi^{2}\right)^{2}$ field theory, we only consider here the CPM symmetrical form by fixing

$$
b_{2}=0
$$

leading to

$$
\Delta \widetilde{n}_{\mathrm{mol}}=\varphi_{1}
$$

The xenon properties of present interest (molar isothermal susceptibility and liquid-gas coexisting molar densities, see below), can be calculated from their dimensionless forms

$$
\begin{aligned}
\left(\widetilde{\chi}_{\mathrm{mol}}\right)^{-1} & =\left(\frac{\partial h_{1}}{\partial \varphi_{1}}\right)_{h_{2}}=\left(\frac{\partial \Delta g^{*}}{\partial \Delta \widetilde{n}_{\mathrm{mol}}}\right)_{T} \\
& =\frac{n_{\mathrm{mol}, c}^{2}}{p_{c}}\left(\frac{\partial \Delta \mu_{\mathrm{mol}}}{\partial \Delta n_{\mathrm{mol}}}\right)_{T}=\frac{n_{\mathrm{mol}, c}^{2}}{p_{c}}\left(\chi_{\mathrm{mol}}\right)^{-1},
\end{aligned}
$$

Equation (22) leads to $\tilde{\chi}_{\mathrm{mol}}=\tilde{\chi}_{\rho}=\left(\frac{\partial \Delta \widetilde{\rho}}{\partial \Delta \widetilde{\mu}}\right)_{T}$ and then to $\tilde{\chi}_{\mathrm{mol}}=\left(Z_{c}\right)^{2} \chi_{\bar{p}}^{*}$. In addition, $\tilde{\chi}_{\mathrm{mol}}=\left(\frac{\partial \varphi_{1}}{\partial h_{1}}\right)_{h_{2}}=\left(\frac{\partial^{2} \Delta \Phi}{\partial h_{1}^{2}}\right)_{h_{2}}=\left(\frac{\partial^{2}\left(p^{*}-1\right)}{\partial\left(\Delta g^{*}\right)^{2}}\right)_{h_{2}}=\frac{p_{c}}{\left(n_{\mathrm{mol}, c}\right)^{2}}\left(\frac{\partial^{2} p}{\partial \mu_{\mathrm{mol}}^{2}}\right)_{T}$ with 
$\left(\frac{\partial p}{\partial \mu_{\mathrm{mol}}}\right)_{T, V=V_{\text {mol }}}=\left(\frac{\partial N}{\partial V}\right)_{T, \mu=\mu_{\mathrm{mol}}}=n_{\mathrm{mol}}(T, p)$. Using the previous relations between densities expressed in units of mole, mass, and particle, the corresponding dimensional susceptibilities are related by $\chi_{\mathrm{mol}}=M_{\mathrm{mol}}^{2} \chi_{\rho}=N_{A}^{2} \chi_{\bar{p}}\left(\right.$ with $\mu_{\mathrm{mol}}=\left(\frac{\partial a}{\partial n_{\mathrm{mol}}}\right)_{T}$ and $\left.\left(\chi_{\mathrm{mol}}\right)^{-1}=\left(\frac{\partial \mu_{\mathrm{mol}}}{\partial n_{\mathrm{mol}}}\right)_{T}=\left(\frac{\partial^{2} a}{\partial n_{\mathrm{mol}}^{2}}\right)_{T}\right)$.

Using our notations for the dimensionless amplitudes and Eqs. (22) and (12), it is thus obtained [16]

$$
\Gamma^{+}=3.38317 m_{0}\left(l_{0}\right)^{-1}
$$

for the leading amplitude of Eq. (12), and

$$
a_{\chi}^{1,+}=g_{\chi}^{+} g^{-\Delta_{s}}(1-\bar{u})
$$

with $g_{\chi}^{+}=0.590$ for the first order amplitude of the related confluent singularities of Eq. (12). We introduce now the asymptotic two-term power law describing the symmetrized top-shape of the liquid-vapor coexistence curve

$$
\Delta \widetilde{\rho}_{L V}=\frac{\rho_{L}-\rho_{V}}{2 \rho_{c}}=B\left|\Delta \tau^{*}\right|^{\beta}\left[1+a_{M}^{1,-}\left|\Delta \tau^{*}\right|^{\Delta}\right]
$$

From Eqs. (21) and (25), it is thus obtained [16]

$$
B=3.28613 m_{0}
$$

for the leading amplitude of Eq. (25), and

$$
a_{M}^{1,-}=g_{M}^{-} g^{-\Delta_{s}}(1-\bar{u})
$$

with $g_{M}^{-}=0.529$ for the first order amplitude of the related confluent singularities of Eq. (25).

In a similar manner, the crossover behavior of the dimensionless correlation length is specified through

$$
\frac{\left(\xi^{*}\right)^{2}}{\widetilde{\chi}_{\text {mol }}}=Y^{-\frac{\eta v}{\Delta_{s}}}(\bar{u} \Lambda)^{-2 \eta v} a\left(\theta, Y_{1}\right)
$$

where $Y_{1}(r)=\frac{1}{\Delta_{s}} \frac{r}{Y} \frac{d Y}{d r}$ and $a\left(\theta, Y_{1}\right)=\left(a_{0}+a_{1} \theta^{2}\right) Y_{1}+\left(a_{0}^{*}+a_{1}^{*} \theta^{2}\right)\left(1-Y_{1}\right)$. The parameters $a_{0}, a_{1}, a_{0}^{*}$, and $a_{1}^{*}$ are the constants that can be determined to reproduce theoretical correlation-length amplitude ratios. They can be expressed with $l_{0}$ and $m_{0}$, as $a_{0}=0.0643\left(l_{0}\right)^{\frac{1}{3}}\left(m_{0}\right)^{-\frac{5}{3}}, a_{1}=0.0178\left(l_{0}\right)^{\frac{1}{3}}\left(m_{0}\right)^{-\frac{5}{3}}, a_{0}^{*}=0.0579\left(l_{0}\right)^{\frac{1}{3}}\left(m_{0}\right)^{-\frac{5}{3}}$, and $a_{1}^{*}=0.0028\left(l_{0}\right)^{\frac{1}{3}}\left(m_{0}\right)^{-\frac{5}{3}}$, leading to

$$
\xi^{+}=0.466287\left(m_{0} l_{0}\right)^{-\frac{1}{3}}
$$

for the leading term amplitude of Eq. (11), and to

$$
a_{\xi}^{1,+}=g_{\xi}^{+} g^{-\Delta_{s}}(1-\bar{u})
$$

with $g_{\xi}^{+}=0.260$ for the first term amplitude of the related confluent singularities of Eq. (11). 
Finally, to carry out the CPM calculation in general, for given reduced temperature and density, the parameters $r$ and $\theta$ are first solved numerically. Then the scaling fields, the thermodynamic potential and its derivatives can be calculated in terms of $r$ and $\theta$ after numerically solving the crossover equation for $Y$.

\subsection{Ising-Like Parameters for the Crossover Formulation of the Parametric EOS}

The generic approach based on a phenomenological crossover transformation for a classical Landau expansion of the singular contribution to a free energy density involves three free parameters, i.e., two asymptotic scaling parameters (noted $c_{t}$ and $c_{\rho}$, in the more general CLM approach) and one crossover parameter (noted $g$ ). Normally, from a field theory framework introducing two asymptotic Ising-like scaling factors, any dimensionless critical crossover description of a 3D Ising-like system with finite cutoff contains at least two other free crossover parameters [4], i.e., a reduced wave number $\Lambda$ related to the cut-off wavelength $\Lambda_{0}$, and a reduced scaling factor $\bar{u}$ related to the coupling constant $u_{4}$. For the theoretical infinite-cutoff approximation in the field theory scheme, $\Lambda \rightarrow \infty, \bar{u} \rightarrow 0$ and $\bar{u} \Lambda$ remains finite. Due to the basic form of the singular part of the free energy density in the CLM description, $g$ is then necessarily related to the product $\bar{u} \Lambda$. However, due to the analytical Landau scheme expressed at the symmetrical fourth-order (with only two independent critical coupling quantities), all the dimensionless quantities are canonical constants (i.e. independent of the selected van der Waals fluid and the dimension of the system). An implicit connection between $\Lambda$ and $\bar{u}$ (or $c_{t}$ ) exists, yielding for example to the explicit relation $g=\frac{(\bar{u} \Lambda)^{2}}{c_{t}}$. Moreover, a single scale unit is needed to reduce dimensional length appearing in correlation and thermodynamic functions, independently of any hypothesis on the microscopic structure of the interacting fluid particles. It also imposes $\Lambda=$ constant for CLM description of the Ising-like systems, leading to a possible arbitrarily choice for the numerical value of the constant provided that $\bar{u} \Lambda$ remains finite. For example, in the case of the 3D-Ising models where $\Lambda=1$, a detailed application [59] of an asymptotic version of CLM has studied the crossover behavior governed by the $\bar{u}$ dependence on the interaction range. This study shows that a more complex, non-monotonic crossover behavior is possible for a very short range of interactions $(\bar{u}>1)$ in complex fluids. In case of the application of the parametric form of the equation of state where $g^{\frac{1}{2}}=\frac{\bar{u} \Lambda}{\left(c_{t}\right)^{\frac{1}{2}}}$ to a one-component fluid such as xenon, the only way to monitor the CLM asymptotic critical crossover, is to change $\bar{u}$, or, equivalently, $c_{t}$, via a single independent coupling quantity (see Ref. [16] for details). In spite of the possible non-universal nature of the resulting complete crossover behavior, such a fluid application offers the opportunity to match the Ising-like singular behaviors predicted by the master crossover functions with the ones of the now called crossover master model $(\mathrm{CMM})$, as described in the following section. This CMM model requiring no adjustable parameters is then for simple fluid systems where $\bar{u}<1$ is strongly correlated with $\Lambda$.

\subsection{Crossover Master Model (CMM)}

The above unknown scaling parameters $l_{0}$ and $m_{0}$ are explicitely involved in the determination of the leading amplitudes of the singular properties, while, similarly, the known scale factors $Y_{c}$ and $Z_{c}$ appear explicitely in the determination of these same leading amplitudes in the master form of the MR scheme. Therefore, to provide the unequivocal link between the $Y_{c} ; Z_{c}$ pair and the $l_{0} ; m_{0}$ pair, we consider the isothermal compressibility and the coexistence curve whose essential role was already mentionned for the singular thermodynamic characterization 
of the fluid. The respective $\Gamma^{+}$and $B$ forms in the master description (see Table I in Ref. [14]) and in the CPM description (see Table III in Ref. [16]), lead to the matching equations:

$$
\Gamma^{+}=\mathcal{Z}_{\chi}^{+} \Lambda_{q e}^{*}\left(Z_{c}\right)^{-1}\left(Y_{c}\right)^{-\gamma}=3.38317 m_{0} l_{0}^{-1}
$$

and

$$
B=\mathcal{Z}_{M}\left(\Lambda_{q e}^{*}\right)^{-1}\left(Z_{c}\right)^{-\frac{1}{2}}\left(Y_{c}\right)^{\beta}=3.28613 m_{0}
$$

The CPM scaling parameters $l_{0}$ and $m_{0}$ can thus be calculated without adjustable parameter using the two following relations,

$$
\begin{array}{r}
l_{0}=\frac{3.38317}{\mathcal{Z}_{\chi}^{+}} \frac{\mathcal{Z}_{M}}{3.28613}\left(\Lambda_{q e}^{*}\right)^{-2}\left(Z_{c}\right)^{\frac{1}{2}}\left(Y_{c}\right)^{\beta+\gamma} \\
m_{0}=\frac{\mathcal{Z}_{M}}{3.28613}\left(\Lambda_{q e}^{*}\right)^{-1}\left(Z_{c}\right)^{-\frac{1}{2}}\left(Y_{c}\right)^{\beta}
\end{array}
$$

Note that Eqs. (33) and (34) also account for quantum effect correction through $\Lambda_{q e}^{*}$ [37] (with $\Lambda_{q e}^{*}=1$ in the non-quantum xenon case).

Moreover, the universality of the first-order correction-to-scaling scheme can also be closed by identifying, for example, the CPM amplitude $\Gamma_{1}^{+}=g_{\chi}^{+} g^{-\Delta_{s}}(1-\bar{u})$ with our selected first-order amplitude $a_{\chi}^{1,+}=\mathcal{Z}_{\varkappa}^{1+}\left(Y_{c}\right)^{\Delta}$ (see Table I in Ref. [14]). Using $g_{\chi}^{+}=$ $0.590, \Delta_{s}=0.52$, and $g=\frac{(\bar{u} \Lambda)^{2}}{c_{t}}$ (see Table III in [16]), we obtain

$$
g_{\chi}^{+}\left[\frac{\bar{u} \Lambda}{\left(c_{t}\right)^{\frac{1}{2}}}\right]^{-2 \Delta_{s}}(1-\bar{u})=\mathcal{Z}_{\varkappa}^{1+}\left(Y_{c}\right)^{\Delta}
$$

Equation (35) demonstrates that the fluid-dependent crossover parameter $g^{\frac{1}{2}}=\frac{\bar{u} \Lambda}{\left(c_{t}\right)^{\frac{1}{2}}}$ introduced in the phenomenological crossover transformation of the Landau expansion is well related to our asymptotic scale parameter $Y_{c}$, as already noted [40] in the case of several non-quantum pure fluids by analyzing the crossover Landau model [17,18]. Equations (33), (34), and (35) are the basic equations which define the master Ising-like nature of the CMM.

\subsection{Comparison of the Calculated Isothermal Compressibility, Coexisting Densities, \\ Correlation Length and Turbidity of Xenon Using CPM and CMM}

Using the arbitrary relation $\frac{\Lambda}{\left(c_{t}\right)^{\frac{1}{2}}}=\pi$ initially adopted by the authors [16], the xenon values of the CPM free parameters $l_{0}, m_{0}$ and $\bar{u}$ reported in column 2 of Table 5 are obtained from a joint fitting of xenon isothermal compressibility (from Eq. (22) and experimental data of Ref. [1]) and xenon coexistence curve (from Eq. (21) and experimental data of Refs. [60,61]). For the CMM case, the calculated values of $l_{0}, m_{0}, \bar{u}$ and $g$ using Eqs. (33), (34), (35) and similar arbitrarily relation $\frac{\Lambda}{\left(c_{t}\right)^{\frac{1}{2}}}=\pi$, are given in column 3 . We note the close agreement between both parameter sets. This satisfactory agreement is confirmed in Figs. 6 and 7 showing the results of the comparison between the experimental data and the two different calculations with free and calculated parameter sets. Finally, the correlation length (from Eq. (23)) and the turbidity (from Eq. (5)) can also be calculated and the corresponding results are illustrated in Fig. 8 where the agreement remains always noticeable. 
Table 5 Adjustable (column 2) and calculated (column 3) values of the Ising-like parameters for CPM and CMM, respectively; References of the experimental data used in the related joint fitting analyses are given in column 4 (see text)

\begin{tabular}{lllc}
\hline Xe & CPM & CMM & Ref. \\
\hline$l_{0}$ & $25.710 \pm 1.128$ & 26.1282 & {$[1]$} \\
$m_{0}$ & $0.4503 \pm 0.0119$ & 0.44665 & {$[60,61]^{\mathrm{a}}$} \\
$\bar{u}$ & $0.1675 \pm 0.067$ & 0.13575 & {$[15]$} \\
$\frac{\Lambda}{\left(c^{\frac{1}{2}}\right.}$ & $\pi($ fixed $)$ & $\pi$ (fixed) & \\
$g^{\frac{1}{2}}$ & $0.526 \pm 0.210$ & 0.41899 & \\
\hline
\end{tabular}

asee also [61]

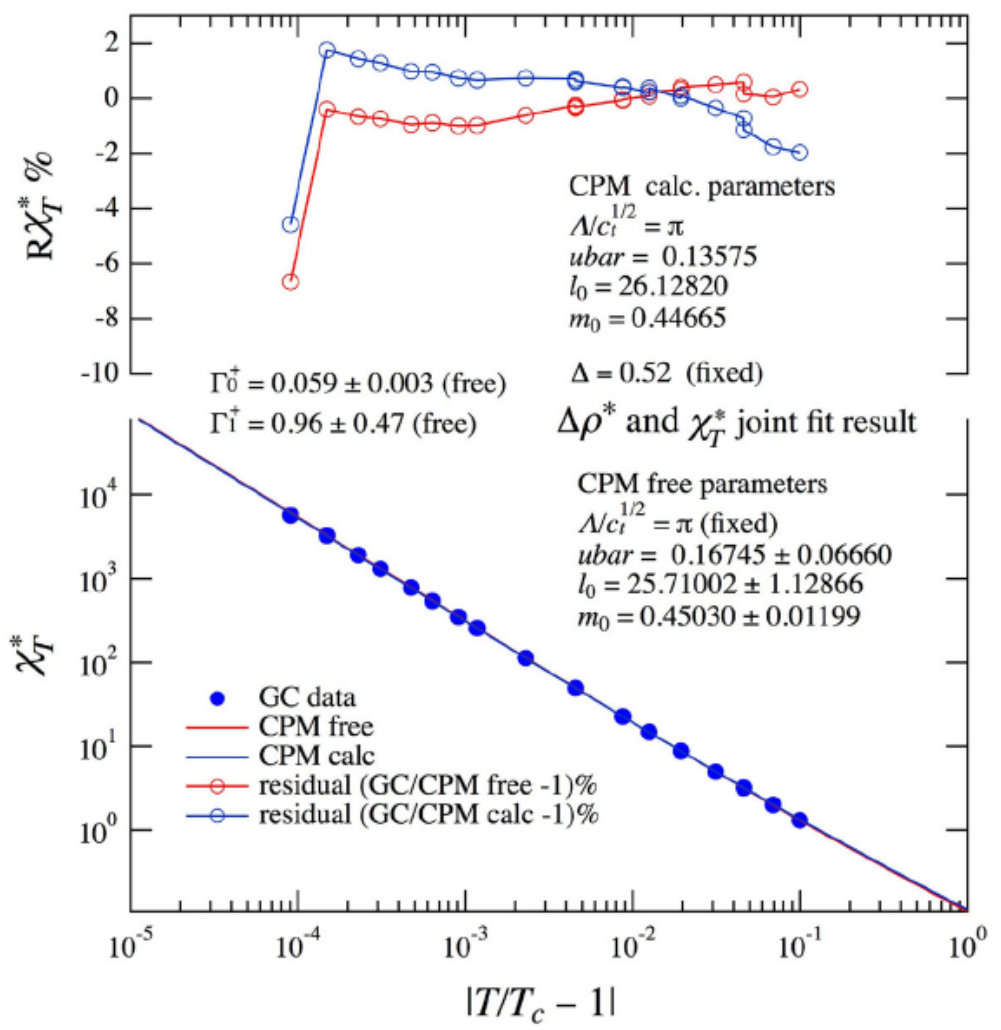

Fig. 6 Log-log scale: Xenon compressibility data (Ref. [1]) is compared with the two cases of CPM model calculations. Corresponding residuals are shown in the upper part (log-linear scale) (Color figure online)

\subsection{Discussion}

The small difference (of order of a few \%) between the estimations of the CPM and MR parameters can be partly due to the numerical differences between the universal features expected from the master form of the MR scheme and from CPM (see Table 2). In the $l_{0}$ and $m_{0}$ cases, the differences are of the same order $(2 \%)$ than the error-bar attached to the direct 


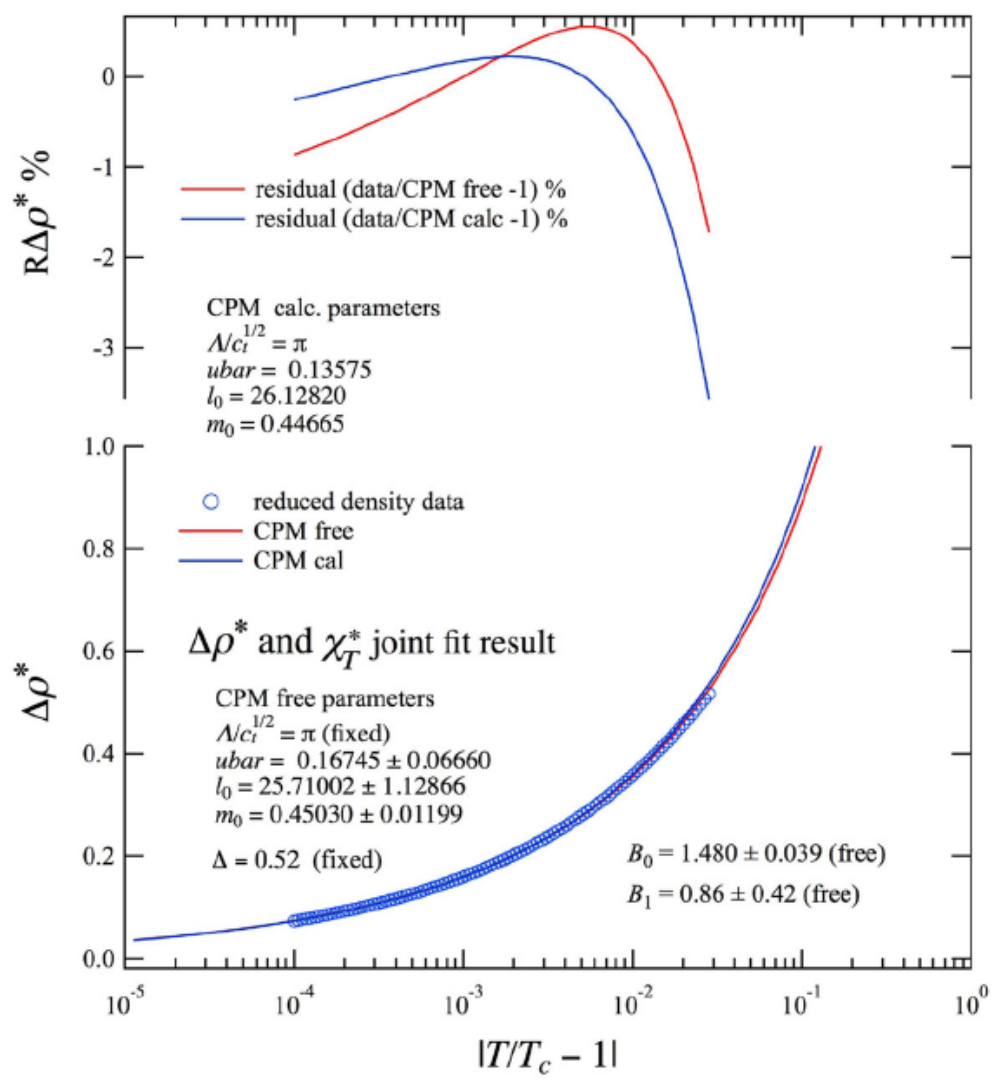

Fig. 7 Log-linear scale: Xenon coexistence curve data (Ref. [60,61]) is compared with the two cases of the CPM model calculations. Corresponding residuals are shown in the upper part (log-linear scale) (Color figure online)

estimation of the leading amplitudes. By using Eq. (35) for the crossover parameter case, we note that the numerical value of the rescaled coupling constant $\bar{u}$ remains dependent on several phenomenological choices of the CPM and master approaches (as for example: the arbitrary relation between $c_{t}, \bar{u}$, and $\Lambda$; the master value $\mathcal{Z}_{\varkappa}^{1+}=0.583$; the numerical differences between universal quantities of the first-order correction-to-scaling scheme). Therefore, the fact that the differences between the free and calculated values of $\bar{u}$ and/or $g^{\frac{1}{2}}$ enter in the error-bar obtained from direct fitting procedure of the joint experimental data must be considered as a satisfactory result.

More generally, we have noted that the expected Ising-like similarity between CMM and the master crossover functions (including the crossover behavior of the correlation length) opens a new route for estimating a complete set of universal parameters only using three of them as readily independent. As CPM, this new Ising-like MR form of CMM offers the main advantage provided by the extended access to the complete phase surface, without any adjustable parameter. It will become a valuable tool to calculate the singular thermophysical properties (including correlation length and turbidity) of any one component fluid, as a function of temperature and density. 

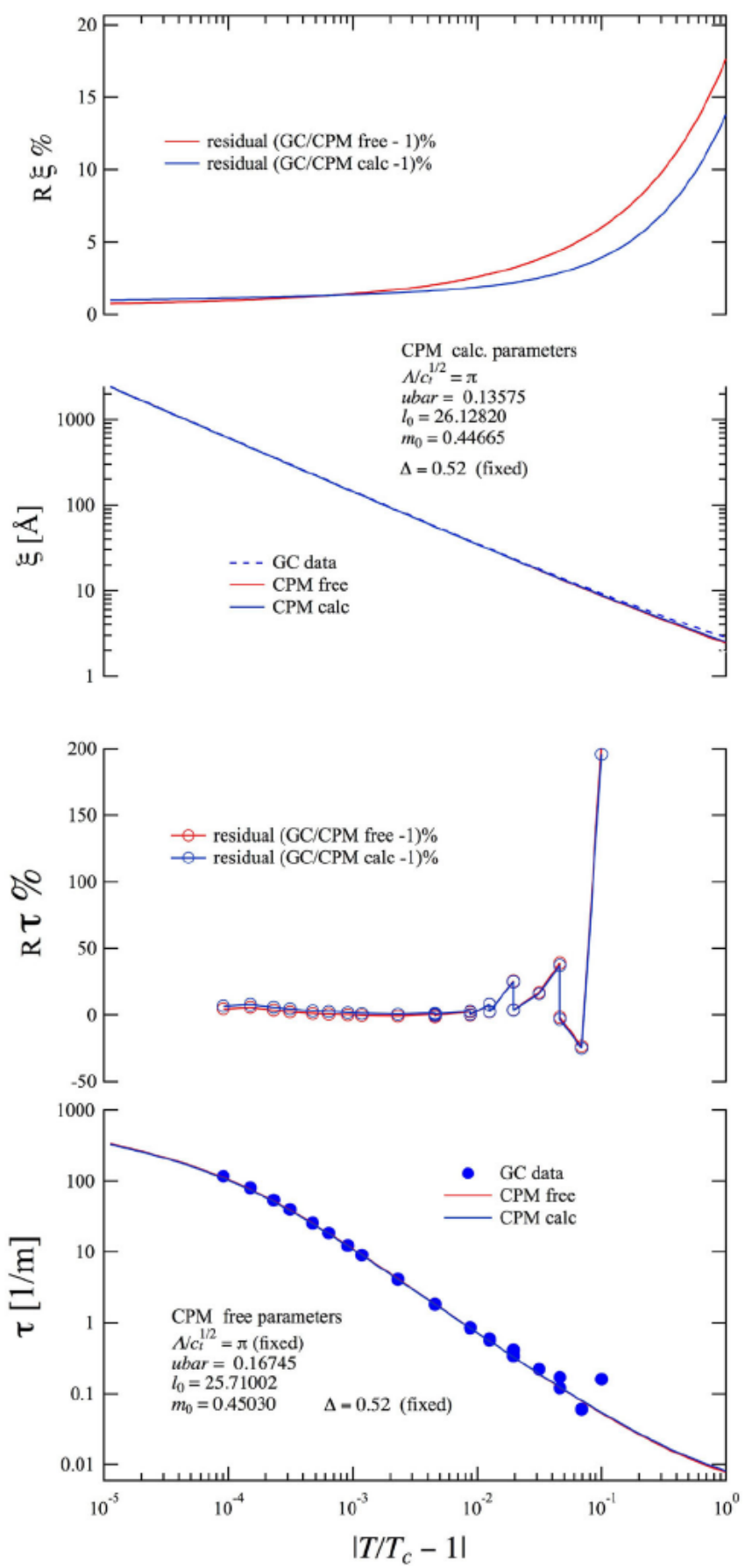

Fig. 8 Log-log scale: GC correlation length data (upper) and GC turbidity data (lower) are compared with the two cases of CPM model calculations. Corresponding residuals are shown in each case (log-linear scale) (Color figure online) 


\section{Conclusion}

Only using the four critical coordinates of the liquid-gas critical point of xenon and the master forms of the Ising-like crossover functions calculated from the massive renormalization scheme for the critical isochore, we have estimated the singular behavior of xenon turbidity in agreement with GC experimental data in the temperature range $28 \mathrm{mK} \leq T-T_{c} \lesssim 9 \mathrm{~K}$ $\left(10^{-4} \lesssim \Delta \tau^{*} \lesssim 3 \times 10^{-2}\right.$ ), contributing then to the expected theoretical progress in the reduction to zero free parameter in the description of the Ising-like confluent singularities far from the critical temperature. The critical xenon, the $N=1$ simple cubic lattice model and the $O(1)$ symmetric $\left(\phi^{2}\right)^{2}$ field theory belong to the same universality class. In addition, we have proposed a crossover master model by calculating the adjustable Ising-like crossover parameters of the crossover parametric model to match the master singular behaviors of the one-components fluids, which opens the route to estimate the singular properties for the near-critical fluids without adjustable parameter.

Acknowledgments We acknowledge discussions and helpful comments from M. A. Anisimov. YG acknowledges F. Palencia for its contribution in the data analyses using Mathematica. The research at ICMCB and PMMH was supported by CNRS and CNES, and at the Jet Propulsion Laboratory, California Institute of Techology, by NASA.

\section{Isothermal Compressibility Data Source}

Before the GC work, the isothermal compressibility data for xenon were generally fitted using the following effective power law

$$
\kappa_{T}^{*}=p_{c} \kappa_{T}=\Gamma_{e}^{+}\left(\Delta \tau^{*}\right)^{-\gamma_{e}}
$$

with adjustable effective (i.e., non-Ising) exponent $\gamma_{e}$ and adjustable effective amplitude $\Gamma_{e}^{+}$. The corresponding $\gamma_{e}$ and $\Gamma_{e}^{+}$values are reported in Table 6 , in addition with the related finite extension $\Delta \tau_{\min }^{*}-\Delta \tau_{\max }^{*}$ and mean position $\left\langle\Delta \tau_{\exp }^{*}\right\rangle=\left(\Delta \tau_{\min }^{*} \Delta \tau_{\max }^{*}\right)^{\frac{1}{2}}$ of the experimental temperature range.

The $\gamma_{e}$-results reported in Table 6 are ordered (see \#i in column 1) from $\gamma$ to $\gamma_{\mathrm{MF}}$, while column 2 indicates the measurement method (FI: Fraunhofer interferometry; LS: light scattering; $p V T:[p(V)]_{T}$ measurements). Data reported in lines \#1a and \#1b are from the interferometric measurements of Hocken and Moldover [50] in the reduced temperature range $10^{-6} \lesssim \Delta \tau^{*} \lesssim 10^{-5}$, using a critical xenon cell subjected to the gravitational field which generates Fraunhofer interferograms [50] related to the isothermal compressibility. The initial fitting with free values of the exponent and amplitude has given $\gamma=1.23$ and $\Gamma^{+}=$ $0.062 \pm 0.006$ [50] (line \#1b), while a subsequent analysis made for fixed Ising value $\gamma=1.24$ has given $\Gamma^{+}=0.058 \pm 0.002$ [51] (line \#1a). Line \#2 is from $\chi_{\rho}=\left(\frac{\partial \Delta \rho}{\partial \Delta \mu_{\rho}}\right)_{T}$ values [62] obtained after numerical integration of $[p(\rho)]_{T}$ data of Habgood and Schneider $[63,64]$ to obtain chemical potential difference $\Delta \mu_{\rho}$ and to validate antisymmetry of the $\left[\Delta \mu_{\rho}(\Delta \rho)\right]_{T}$ supercritical isotherms (and then symmetry of $\left[\chi_{\rho}(\Delta \rho)\right]_{T}$ ), as functions of $\Delta \rho=\rho-\rho_{c}$ (see also Footnote 5). Data reported in line \#3 are from dynamic light scattering data and complex analysis of the xenon Brillouin spectra by Cannell and Benedek [43] covering the temperature range $0.2 \mathrm{~K} \leq T-T_{c} \leq 20 \mathrm{~K}$. Data reported in line \#4 are from static light scattering data of Smith et al. [42] covering the temperature range $0.045 \mathrm{~K} \leq T-T_{c} \leq 5.1 \mathrm{~K}$. After calibration of the isothermal compressibility at $T=T_{c}+1 K$ [66], the effective amplitude for Cannell 
Table $6 \gamma_{e}$ (column 3) and $\Gamma_{e}$ (column 4) values published before the date of I, from $\kappa_{T}^{*}=\Gamma_{e}^{+}\left(\Delta \tau^{*}\right)^{-\gamma_{e}}$ fitting in a restricted temperature range $\Delta \tau_{\min }^{*} \leq \Delta \tau^{*} \leq \Delta \tau_{\max }^{*}$ along the critical isochore of xenon for $T>T_{C}\left(()^{\star}\right.$ corresponds to a fixed value in the fit)

\begin{tabular}{|c|c|c|c|c|c|c|c|}
\hline \# & & $\gamma_{e}$ & $\Gamma_{e}^{+}$ & $\Delta \tau_{\min }^{*}$ & $\Delta \tau_{\max }^{*}$ & $\left\langle\Delta \tau_{\exp }^{*}\right\rangle$ & Ref. \\
\hline $1 a$ & FI & $\left(\gamma_{\text {Ising }}=1.24\right)^{\star}$ & $0.058 \pm 0.002$ & $10^{-6}$ & $10^{-5}$ & $3 \times 10^{-6}$ & {$[50,51]$} \\
\hline $1 b$ & FI & 1.23 & $0.062 \pm 0.006$ & $10^{-6}$ & $10^{-5}$ & $3 \times 10^{-6}$ & [50] \\
\hline 2 & $p V T$ & $1.211( \pm 0.025)$ & $0.0743( \pm 0.015)$ & $6.0 \times 10^{-4}$ & $6.0 \times 10^{-3}$ & $1.9 \times 10^{-3}$ & [62-64] \\
\hline 3 & LS & 1.21 & 0.076845 & $6.9 \times 10^{-4}$ & $6.9 \times 10^{-2}$ & $6.9 \times 10^{-3}$ & [43] \\
\hline 4 & LS & $1.21 \pm 0.03$ & 0.06742 & $1.553 \times 10^{-4}$ & $1.76 \times 10^{-2}$ & $1.653 \times 10^{-3}$ & [42] \\
\hline 5 & LS & 1.206 & 0.07551466 & $9.6 \times 10^{-5}$ & $1.0 \times 10^{-1}$ & $4.35 \times 10^{-3}$ & I and [15] \\
\hline 6 & $p V T$ & $\left(\gamma_{e, \text { eos }}=1.190\right)^{\star}$ & 0.0793 & $5.0 \times 10^{-4}$ & $3.0 \times 10^{-2}$ & $3.873 \times 10^{-3}$ & {$[63-65,67]$} \\
\hline 7 & $p V T$ & 1.1665 & 0.089 & $6.2 \times 10^{-3}$ & $8.1 \times 10^{-2}$ & $2.24 \times 10^{-2}$ & [11,67-72] \\
\hline 8 & $p V T$ & $\left(\gamma_{\frac{1}{2}}=1.12\right)^{\star}$ & 0.10 & $10^{-1}$ & $2.9 \times 10^{-1}$ & $1.70 \times 10^{-1}$ & [66-72] \\
\hline 9 & $p V T$ & $\left(\gamma_{\mathrm{MF}}=1\right)^{\star}$ & 0.11 & 0.38 & 0.72 & $5.2 \times 10^{-1}$ & {$[66,68-72]$} \\
\hline
\end{tabular}

\# (column 1): ordered number of the segment in Fig. 1a (see text). Measurement method (column 2): $p V T$ : $[p(V)]_{T}$ analysis; LS: light scattering; FI: Fraunhofer interferometry. $\Delta \tau_{\min ^{*}}^{*}, \Delta \tau_{\max }^{*}$ and $\left\langle\Delta \tau_{\exp }^{*}\right\rangle-$ values (columns 5 to 7, respectively); References (column 8)

and Benedek's data was lowered by $1.57 \%$, while the one for Smith et al's data was increased by $11.2 \%$, in agreement with around $\simeq 14 \%$ initial deviation between these two data set. In spite of the importance $(\simeq(10-20) \%)$ of these amplitude corrections, the fitting results confirm that the $\gamma_{e}$ value is well in the range $\gamma_{e} \simeq 1.20-1.21$ for the restricted temperature range of these experiments. Such results were confirmed by the data in line \#5 obtained by the effective power law fitting of the GC's measurements of high relative precision in the similar temperature range. As a matter of fact, GC have claimed that the correction to scaling terms are important by demonstrating that the susceptibility deviates systematically from Eq. 36 with $\gamma_{e}=1.206$. More precisely, Fig. 2 of I shows that $\gamma_{e} \simeq 1.206$ is the slope of the tangent line to the rough experimental behavior of $\kappa_{T}^{*}\left(\Delta \tau^{*}\right)$ at $\Delta \tau_{\gamma_{e}=1.206}^{*} \simeq 4.35 \times 10^{-3}$, i.e., the temperature distance which corresponds to the minimum of the deviation curve in this Fig. 2 (see also Fig. 5 in Ref. [15]). The finite value $\Delta \tau_{\gamma_{e}=1.206}^{*}$ is clearly outside the PAD extension (see the related circle in the segment 5 of Fig. 1a).

Now we note that the line \#6 in Table 6 is also from directly fitting $[65]^{3}[p(V)]_{T}$ measurements of Habgood and Schneider [63,64] and Michels et al. [67], using the wellknown restricted linear model of the scaled "universal" equation of state, where $k(\mathrm{Xe})=$ 1.309, $a(\mathrm{Xe})=16.1$ and $\gamma_{e, \text { eos }}=1.190$ [65], leading to $\Gamma_{e}^{+}=\frac{k}{a}$. In addition, the lines \#7 to \#9 are from similar $p V T$ analyses [66] using the $[p(V)]_{T}$ data measurements of Michels et al. [67], Beattie et al. [68,69], and Rabinovich et al. [70-72]. Increasing thus the covered temperature distance to $T_{c}$ leads to observe the continuous decrease of $\gamma_{e}$ in the range $2 \times 10^{-2} \lesssim \Delta \tau^{*} \lesssim 1$ (see the corresponding segments 6 to 9 in Fig. 1a). Practically, the $\Delta \tau^{*}$ range where $\gamma_{e} \simeq \gamma_{\mathrm{MF}}=1$ starts in the well-defined temperature range $400<$ $T(K)<500$ [66]. Therefore, the physical mean field limit $\Delta \tau^{*} \gtrsim 1$ is reached after crossing down the effective mean value $\gamma_{\frac{1}{2}}=\frac{\gamma+\gamma_{\mathrm{MF}}}{2} \simeq 1.12$ around $\Delta \tau^{*} \simeq 0.14$ [see Fig.

${ }^{3}$ See Table 4.3.4, p. 144 in reference [65]. 
1a] and by following an abrupt $\gamma_{e}$ decrease to $\gamma_{e} \simeq 1$, over a well-descrimined sharp domain $0.3 \leq \Delta \tau_{\mathrm{CO}}^{*} \leq 0.5$ (see the double arrow in Fig. 1b). In Fig. 1a, this mean-field-like side of the physical crossover is illustrated by the interpolating line labelled CO from $\gamma_{\frac{1}{2}}$ in the MR curve and $\gamma_{e} \simeq \gamma_{\mathrm{MF}}=1$ at $\left\langle\Delta \tau_{\exp }^{*}\right\rangle \simeq 0.5$ in the segment 9 . However, the predicted mean-field-like side from the theoretical crossover function (right part of the figure) is not compatible with these experimental results, which means that the physical crossover (curve $\mathrm{CO}$ ) is not accounted for by the MR approach (curve MR) of the classical-to-critical crossover (see also Ref. [12,13]).

\section{References}

1. Güttinger, H., Cannell, D.S.: Corrections to scaling in the susceptibility of xenon. Phys. Rev. A 24, 3188-3201 (1981)

2. Anisimov, M.A., Sengers, J.V.: 11 Critical region. In: Sengers, J.V., Kayser, R.F., Peters, C.J., White Jr, H.J. (eds.) Equations of State for Fluids and Fluid Mixtures, pp. 381-434. Elsevier, Amsterdam (2000)., part I, and references therein

3. Zinn-Justin, J.: Quantum Field Theory and Critical Phenomena, 4th edn. Clarendon, Oxford (2002)

4. Bagnuls, C., Bervillier, C.: Classical-to-critical crossovers from field theory. Phys. Rev. E 65, 066132-12p (2002)

5. Garrabos, Y., Bervillier, C.: Mean crossover functions for uniaxial 3D Ising-like systems. Phys. Rev. E 74, 021113-16p (2006)

6. Guida, R., Zinn-Justin, J.: Critical exponents of the N-vector model. J. Phys. A : Math. Gen. 31, 8103-8122 (1998)

7. Privman, V., Hohenberg, P.C., Aharony, A.: Universal critical point amplitude relations. In: Domb, C., Lebowitz, J. (eds.) Phase Transitions and Critical Phenomena, vol. 14, pp. 1-134. Academic Press, New York (1991)

8. Wegner, F.J.: Corrections to scaling laws. Phys. Rev. B 5, 4529-4536 (1972)

9. Garrabos, Y.: Facteurs d'échelle phénoménologiques pour la transition critique liquide-gaz des fluides purs. J. Phys. (Paris) 46, 281-291 (1985)

10. Garrabos, Y.: see also: Phenomenological Scale Factors for the Liquid-Vapor Critical Transition of Pure Fluids. cond-mat/0512408

11. Garrabos, Y.: Scaling behaviour of the fluid subclass near the liquid-gas critical point. J. Phys. (Paris) 47, 197-206 (1986)

12. Bagnuls, C., Bervillier, C.: Nonasymptotic critical behaviour from field theory for Ising like systems in the homogeneous phase: theoretical framework. J. Phys. (Paris) Lett. 45, L-95-L-100 (1984)

13. Bagnuls, C., Bervillier, C., Garrabos, Y.: Experimental data analysis on xenon above the critical temperature from nonlinear renormalization group. J. Phys. (Paris) Lett. 45, L-127-L-132 (1984)

14. Garrabos, Y., Lecoutre, C., Palencia, F., LeNeindre, B., Erkey, C.: Master crossover functions for onecomponent fluids. Phys. Rev. E 77, 021116-26p (2008)

15. Garrabos, Y., Lecoutre, C.: Characteristic parameters of xenon near its liquid-gas critical point, preprint21p. (2009); see hal-00363930

16. Agayan, V.A., Anisimov, M.A., Sengers, J.V.: Crossover parametric equation of state for Ising-like systems. Phys. Rev. E 64, 026125-19p (2001)

17. Chen, Z.Y., Albright, P.C., Sengers, J.V.: Crossover from singular critical to regular classical thermodynamic behavior of fluids. Phys. Rev. A 41, 3161-3177 (1990)

18. Chen, Z.Y., Abbaci, A., Tang, S., Sengers, J.V.: Global thermodynamic behavior of fluids in the critical region. Phys. Rev. A 42, 4470-4484 (1990)

19. Nicoll, J.F., Bhattacharjee, J.K.: Crossover functions by renormalization-group matching: $O\left(\epsilon^{2}\right)$ results. Phys. Rev. B 23, 389-401 (1981)

20. Nicoll, J.F., Albright, P.C.: Crossover functions by renormalization-group matching: three-loop results. Phys. Rev. B 31, 4576-4589 (1985)

21. Nicoll, J.F., Albright, P.C.: Background fluctuations and Wegner corrections. Phys. Rev. B 34, 1991-1996 (1986)

22. Ornstein, L.S., Zernike, F.: Accidental deviations of density and opalescence at the critical point of single substance. Proc. Acad. Sci. Amst. 17, 793-806 (1914)

23. Ornstein, L.S., Zernike, F.: The linear dimension of density variations. Phys. Z 19, 134-137 (1918) 
24. Puglielli, V.G., Ford, N.C.: Turbidity measurements in SF6 near its critical point. Phys. Rev. Lett. 25, 143-146 (1970)

25. Fabelinskii, L.: I. Molecular Scattering of Light. Plenum Press, New York (1968)

26. Levelt-Sengers, J.M.H.: Scaling predictions for thermodynamic anomalies near the gas-liquid critical point. Ind. Eng. Chem. Fundam. 9, 470-480 (1970)

27. Garrabos, Y., Lecoutre, C., Palencia, F., Broseta, D., LeNeindre, B.: Master crossover behavior of parachor correlations for one-component fluids. Phys. Rev. E 76, 061109-22p (2007)

28. Green, M.S.: On the theory of the critical point of a simple fluid. J. Chem. Phys. 33, 1403-1409 (1960)

29. Fisher, M.E.: Correlation functions and the critical region of simple fluids. J. Math. Phys. 5, 944-962 (1964)

30. Bray, A.J.: Predicted critical correlation function for three-dimensional phase transitions. Phys. Rev. Lett. 36, 285-288 (1976)

31. Bray, A.J.: Dispersion-theory approach to the correlation function for critical scattering. Phys. Rev. B 14, 1248-1270 (1976)

32. Ferrell, R.A.: Determination of the anomalous dimension Green-Fisher critical exponent $\eta$ by light scattering in a fluid. Phys. A 177, 201-206 (1991)

33. Martin-Mayor, V., Pelissetto, A., Vicari, E.: Critical structure factor in Ising systems. Phys. Rev. E 66, 026112-9p (2002)

34. Chapman, J.A., Finnimore, P.C., Smith, B.L.: Temperature and density dependence of the molar polarizability of xenon. Phys. Rev. Lett. 21, 1306-1307 (1968)

35. Garside, D.H., Molgaard, H.V., Smith, B.L.: Refractive index and Lorentz-Lorenz function of xenon liquid and vapour. J. Phys. B: Phys. Soc. (London) Proc. 1, 449-457 (1968)

36. Gillis, K.A., Shinder, I.I., Moldover, M.R.: Thermoacoustic boundary layers near the liquid-vapor critical point. Phys. Rev. E 70, 021201-20p (2004)

37. Garrabos, Y.: Universality and quantum effects in one-component critical fluid. Phys. Rev. E 73, 056110$13 p(2006)$

38. Kouvel, J.S., Fisher, M.E.: Detailed magnetic behavior of nickel near its curie point. Phys. Rev 136, A1626-A1632 (1964)

39. Hirschfelder, J.O., Curtiss, C.F., Bird, R.B.: Molecular Theory of Gases and Liquids. Wiley, New York (1954)

40. Garrabos, Y., Lecoutre, C., Palencia, F., Erkey, C., LeNeindre, B.: Master singular behavior from correlation length measurements for seven one-component fluids near their gas-liquid critical point. Phys. Rev. E 73, 026125-9p (2006)

41. Garrabos, Y., Lecoutre, C., Marre, S., Guillaument, R., Beysens, D., Hahn, I.: Test of Ising-like equationof-state models using turbidity measurements in near critical fluids, Preprint, 18 p (2013)

42. Smith, I.W., Giglio, M., Benedek, G.B.: Correlation range and compressibility of xenon near the critical point. Phys. Rev. Lett. 27, 1556-1559 (1971)

43. Cannell, D.S., Benedek, G.B.: Brillouin spectrum of xenon near its critical point. Phys. Rev. Lett. 25, $1157-1161(1970)$

44. Zollweg, J., Hawkins, G., Smith, I.W., Giglio, M., Benedek, G.B.: The spectrum and intensity of light scattered from the bulk phases and from the liquid-vapor interface of xenon near its critical point. J. Phys. (Paris) 33, C1-135-139 (1972)

45. Swinney, H.L., Henry, D.L., Cummins, H.Z.: The Rayleigh linewidth in xenon near the critical point. J. Phys. (Paris) 33, C1-81-90 (1972)

46. Swinney, H.L., Henry, D.L.: Dynamics of fluids near the critical point: decay rate of order-parameter fluctuations. Phys. Rev. A 8, 2586-2617 (1973)

47. Güttinger, H., Cannell, D.S.: Correlation range and Rayleigh linewidth of xenon near the critical point. Phys. Rev. A 22, 285-286 (1980)

48. Berg, R.F., Moldover, M.R., Zimmerli, G.A.: Frequency-dependent viscosity of xenon near the critical point. Phys. Rev. E 60, 4079-4098 (1999)

49. Gillis, K.A., Shinder, I.I., Moldover, M.R.: Bulk viscosity of stirred xenon near the critical point. Phys. Rev. E 72, 051201-20p (2005)

50. Hocken, R., Moldover, M.R.: Ising critical exponents in real fluids: an experiment. Phys. Rev. Lett. 37, 29-32 (1976)

51. Sengers, J.V., Moldover, M.R.: Two-scale-factor universality near the critical point of fluids. Phys. Lett. 66A, 44-46 (1978)

52. Moldover, M.R., Sengers, J.V., Gammon, R.W., Hocken, R.J.: Gravity effects in fluids near the gas-liquid critical point. Rev. Mod. Phys. 51, 79-99 (1979)

53. Berg, R.F., Lyell, M.J., McFadden, G.B., Rehm, R.G.: Internal waves in xenon near the critical point. Phys. Fluids 8, 1464-1475 (1996) 
54. Anisimov, M.A., Povodyrev, A.A., Kulikov, V.D., Sengers, J.V.: Nature of crossover between Ising-like and mean-field critical behavior in fluids and fluid mixtures. Phys. Rev. Lett. 75, 3146-3149 (1995)

55. Luijten, E., Meyer, H.: Crossover behavior in $3 \mathrm{He}$ and Xe near their liquid-vapor critical point. Phys. Rev. E 62, 3257-3261 (2000)

56. Hahn, I., Zhong, F., Barmatz, M., Haussmann, R., Rudnick, J.: Crossover behavior in the isothermal susceptibility near the 3 He critical point. Phys. Rev. E 63, 055104(R)-4p (2001)

57. Bagnuls, C., Bervillier, C., Meiron, D.I., Nickel, B.G.: Non-asymptotic critical behavior from field theory at d=3. II. The ordered phase case. Phys. Rev. B 35, 3585-3607 (1987)

58. Fisher, M.E., Zinn, S.-Y.: The shape of the van der Waals loop and universal critical amplitude ratios. J. Phys. A: Math. Gen. 31, L629-L636 (1998)

59. Kim, Y.C., Anisimov, M.A., Sengers, J.V., Luitjen, E.: Crossover critical behavior in the three-dimensional Ising model. J. Stat. Phys. 110, 591-609 (2003)

60. Närger, U., Balzarini, D.A.: Coexistence-curve diameter and critical density of xenon. Phys. Rev. B 42, 6657 (1990)

61. Balzarini, D., Mouritsen, O.G.: Universal ratio of correction-to-scaling amplitudes for Xe. Phys. Rev. A 28, 3515-3519 (1983)

62. Levelt Sengers, J.M.H., Greer, W.L., Sengers, J.V.: Scaled equation of state parameters for gases in critical region. J. Phys. Chem. Ref. Data 5, 1-51 (1976)

63. Habgood, H.W., Schneider, W.G.: PVT measurements in the critical region of xenon. Can. J. Chem. 32, 98-112 (1954)

64. Habgood, H.W., Schneider, W.G.: Thermodynamic properties of xenon in the critical region. Can. J. Chem. 32, 164-173 (1954)

65. Levelt Sengers, J.M.H., Sengers, J.V.: Critical phenomena in classical fluids, chap. 4. In: Croxton, C.A. (ed.) Progress in Liquid Physics, pp. 103-174. Wiley, New York (1978)

66. Garrabos, Y.: Contribution à l'étude des propriétés d'état des fluides purs dans leur région critique, Thése de Doctorat d'Etat, Université Paris 6 (1982)

67. Michels, A., Wassenaar, T., Louwerse, P.: Isotherms of xenon at temperatures between $0 \mathrm{C}$ and $150 \mathrm{C}$ and at densities up to 515 Amagats (pressures up to 2800 atmospheres). Physica 20, 99-106 (1954)

68. Beattie, J.A., Barriault, R.J., Brierley, J.S.: The compressibility of gaseous xenon. I. An equation of state for xenon and the weight of a liter of xenon. J. Chem. Phys. 19, 1219-1221 (1951)

69. Beattie, J.A., Barriault, R.J., Brierley, J.S.: The compressibility of gaseous xenon. II. The virial coefficients and potential parameters of xenon. J. Chem. Phys. 19, 1222-1226 (1951)

70. Rabinovich, V.A., Tokina, L.A., Berezin, V.M.: Experimental determination of the compressibility of krypton and xenon in the 300-720 K range and up to 400 bars. Teplofiz. Vysok. Temp. 11, 64-73 (1973)

71. Rabinovich, V.A., Abovskii, V.A.: Equation of state of a monoatomic liquid at high densities. Teplofiz. Vysok. Temp. 8, 46-54 (1970)

72. Abovskii, V.A., Rabinovich, V.A.: Cell model for monoatomic gases and liquids. Private prepint paper (in Russia) (1973) 